\title{
The Elite Alleles of OsSPL4 Regulate Grain Size and Increase Grain Yield in Rice
}

\author{
Jihong Hu ${ }^{1,2,3+}{ }^{\infty}$, Liyu Huang ${ }^{4 \dagger}$, Guanglong Chen ${ }^{1 \dagger}{ }^{*}$ Hui Liu $^{5 \dagger}$, Yesheng Zhang ${ }^{2,6}$, Ru Zhang ${ }^{3}$, Shilai Zhang ${ }^{4}$,

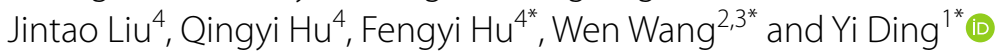

\begin{abstract}
Grain weight and grain number, the two important yield traits, are mainly determined by grain size and panicle architecture in rice. Herein, we report the identification and functional analysis of OSSPL4 in panicle and grain development of rice. Using CRISPR/Cas9 system, two elite alleles of OSSPL4 were obtained, which exhibited an increasing number of grains per panicle and grain size, resulting in increase of rice yield. Cytological analysis showed that OsSPL4 could regulate spikelet development by promoting cell division. The results of RNA-seq and qRT-PCR validations also demonstrated that several MADS-box and cell-cycle genes were up-regulated in the mutation lines. Co-expression network revealed that many yield-related genes were involved in the regulation network of OSSPL4. In addition, OSSPL4 could be cleaved by the osa-miR156 in vivo, and the OsmiR156-OsSPL4 module might regulate the grain size in rice. Further analysis indicated that the large-grain allele of OSSPL4 in indica rice might introgress from aus varieties under artificial selection. Taken together, our findings suggested that OsSPL4 could be as a key regulator of grain size by acting on cell division control and provided a strategy for panicle architecture and grain size modification for yield improvement in rice.
\end{abstract}

Keywords: OsSPL4, Grain size, Grain weight, Panicle branching, Grain yield, Rice

\section{Introduction}

Rice (Oryza sativa L.) is a staple food for more than half of the world's population. Increasing grain yield is a longterm goal for crop breeding to meet the demand of global food security. At the individual plant level, rice grain yield is determined by three component traits: number of tillers (panicles) per plant, number of grains per panicle, and grain weight (Mao et al. 2010). Panicle length,

\footnotetext{
*Correspondence: hfengyi@ynu.edu.cn; wwang@mail.kiz.ac.cn; yiding@whu. edu.cn

${ }^{\dagger}$ Jihong Hu, Liyu Huang, Guanglong Chen and Hui Liu have contributed equally to this work

${ }^{1}$ State Key Laboratory of Hybrid Rice, College of Life Sciences, Wuhan University, Wuhan 430072, China

2 State Key Laboratory of Genetic Resources and Evolution, Kunming Institute of Zoology, Chinese Academy of Sciences, Kunming 650223, China

${ }^{4}$ State Key Laboratory for Conservation and Utilization of Bio-Resources in Yunnan, School of Agriculture, Yunnan University, Kunming 650500,

China

Full list of author information is available at the end of the article
}

the number of panicle branches and the number of spikelets per panicle are the components of panicle architecture (Bai et al. 2017). In rice, grain weight is controlled by grain size which includes grain length, grain width and grain thickness (Zuo and Li 2014; Hu et al. 2015b). Grain size is a key determinant of grain yield and is also a target trait during domestication and breeding in rice (Xia et al. 2018).

In the past years, many genes and quantitative trait loci (QTLs) controlling panicle architecture and grain size have been cloned and characterized (Bai et al. 2012; Zou and $\mathrm{Li}, 2014$; Li et al. 2019). A few genes for panicle architecture have been well studied, such as OsGRF6, OsSPL14 and OsLG1, which could promote panicle branching or form compact panicle (Gao et al. 2015; Miura et al. 2010; Zhu et al. 2013). For grain size, several genes, including DEP1, GS3, GL3.1, GL7, GL3.3/TGW3 and OsGRF4, have been reported to regulate grain length through coordinating alternation of cell division and expansion (Mao 
et al. 2010; Qi et al. 2012; Wang et al. 2015; Sun et al. 2016; Xia et al. 2018; Ying et al. 2016). And other genes such as GW2, qSW5/GW5, GS5, GS6, GW7 and GW8 regulate grain width via activation of cell division (Song et al. 2007; Weng et al. 2008; Li et al. 2011; Wang et al. 2012; Sun et al. 2013; Liu et al. 2017). Plant hormones (brassinosteroid and auxin) have also been reported to directly or indirectly participate in controlling rice grain size ( $\mathrm{Hu}$ et al. 2018b; $\mathrm{Li}$ and $\mathrm{Li}, 2016$ ). For example, qGL3/OsPPKL1, GS2/GL2, GS5 and GW5 are involved in brassinosteroid (BR) signaling pathway (Weng et al. 2008; Li et al. 2011; Zhang et al. 2012; Che et al. 2015; Liu et al. 2017), while $T G W 6$ and $B G 1$ are induced by auxin (Ishimaru et al. 2013; Liu et al. 2015). However, only several genes affect both panicle architecture and grain size, including OsmiR397-OsLAC, FZP and OsSPL13 (Zhang et al. 2013; Si et al. 2016; Bai et al. 2017). Although a number of QTLs/genes for panicle architecture and grain size have been cloned, more genes are needed to understand the genetic and molecular basis of rice yield. Therefore, more genes controlling these important traits remain to be identified for further improvement of yield in rice.

As a class of plant-specific transcription factors, the SQUAMOSA PROMOTER BINDING PROTEIN (SBP)like (SPL) family proteins all contain a highly conserved DNA-binding domain (SBP domain) of 76 amino acids, harboring a nuclear localization signal at its $\mathrm{C}$-terminus, which is sufficient to bind DNA contained two zinc-fingers of a unusual structure (Birkenbihl et al. 2005; Xie et al. 2006; Yamasaki et al. 2006). It has been proved that the DNA binding domain (DBD) of SBP-box genes is necessary and sufficient to bind a palindromic GTAC core motif (Birkenbihl et al. 2005; Guo et al. 2008). In addition, some studies have demonstrated that the miR156SPL module plays important roles in plant growth and development (Wang and Zhang 2017; Dai et al. 2018). Recent studies showed that SPL family genes regulate the panicle architecture or grain size, thus affecting the grain yield in rice (Jiao et al. 2010; Wang et al. 2012; Si et al. 2016). Fine-tuning the expression of SPL may provide a strategy for increasing grain productivity in rice breeding (Wang and Zhang 2017).

In rice, a total of $19 S P L$ genes were identified as six subgroups. Many of these genes are located within segmental duplication regions, including the gene pairs of OsSPL3/12, OsSPL4/11, OsSPL14/17 and so on (Xie et al. 2006; Zhong et al. 2019). Several OsSPL genes (OsSPL13, OsSPL14, OSSPL16 and OSSPL18) have been cloned and reported to regulate panicle development or grain size as well as grain shape and quality in rice (Jiao et al. 2010; Miura et al. 2010; Wang et al. 2012, 2018; Si et al. 2016). On the other hand, OsSPL8/OsLG1 controls the compact panicle architecture in domesticated rice cultivars (Zhu et al. 2013). Moreover, OsSPL6 can repress the endoplasmic reticulum (ER) stress signaling outputs to prevent the occurrence of panicle apical abortion (Wang et al. 2018). OsSPL7 has been reported to regulate tiller number and plant height via miR156f-OsSPL7-OsGH3.8 pathway (Dai et al. 2018). In addition, OsSPL9 can mediate the transcriptional activation of miR528 and orchestrates the antiviral response in rice (Yao et al. 2019). Lately, OsSPL3/OsSPL12 were reported to directly activate OsMADS50 in the node, which regulates the crown root development in rice (Shao et al. 2019). In addition, comprehensive functional assessments of the OsSPL gene family in rice preliminary revealed some of them regulate grain size with mutants using CRISPR/Cas9 (Jiang et al., 2020). However, the biological function of other OsSPLs, including OSSPL4 in rice is still limited.

OsSPL4 and OSSPL11 are a duplicated gene pair, but the last exon of OsSPL4 is shorter than that of OsSPL11. Most of the rice OSSPL genes possessed a unique motif which could be targeted by OsmiR156, but the miRNA target site of OsSPL4 was located in the 3'-UTR, which is similar to OsSPL13 (Xie et al. 2006; Si et al. 2016). In Arabidopsis, the OsSPL4 homologue genes, AtSPL2, AtSPL10 and AtSPL11 have been reported to control the development of lateral organs associated with shoot maturation in the reproductive phase (Shikata et al. 2009). Lately, systematic biology analysis revealed that OsSPL4 might affect panicle development and grain weight to enhance yield in rice (Hu et al., 2020; Jiang et al., 2020). However, the biological function, artificial selection and evolution of OSSPL4 in rice have not been well characterized.

Here, we reported the molecular and genetic analysis of OSSPL4 and revealed some important structural and functional features as well as the evolution of the OSSPL4 in panicle architecture and grain size regulation of rice. Our results suggested that OsSPL4 plays multiple roles in panicle architecture, grain size and yield in rice.

\section{Materials and Methods \\ Plant Materials and Growth Conditions}

The Nipponbare rice (Oryza sativa L. subsp. japonica. cv. Nipponbare) and different independent homozygous CRISPR/Cas9 mutation lines of T3 generation were used in this study. Meanwhile, the empty vector with Nipponbare background was used as the control. Using CRISPR-P (http://crispr.hzau.edu.cn/CRISPR2/), a 20 bp target site (5'-AGGTGCCAGGTGGAAGGGTG-3') upstream of the protospacer-adjacent motif (PAM) was chosen for OSSPL4 (LOC_Os02g07780) gene editing with the CRISPR/Cas9 system. The target vectors were constructed using CRISPR/Cas9 binary vector pCAMBIA1300-OsU3-Cas9 by the restriction enzyme Aar I as described (Huang et al. 2018). Among the transgenic 
plants of the T0 generation, the various mutations of OsSPL4 were confirmed by sequencing.

To generate transgenic rice plants by overexpressing of OSSPL4 and OsmiR156, the coding region from first-strand cDNA of OsSPL4, and the genomic DNA sequences of pre-miR156 was amplified and cloned, respectively. The PCR product was then inserted into a pBWA vector along with the maize UBIQUITIN (Ubi) promoter and Nos terminator. To efficient degradation of OsmiR156, the short tandem target mimic (STTM) of STTM156k was constructed as previous described (Yan et al. 2012). The primer sequences for these constructs are listed in Additional file 1: Table S1.

The vectors were transferred into EH105, a strain of Agrobacterium tumefaciens by electroporation. The calli derived from cultivar Nipponbare rice were used for Agrobacterium-mediated transformation (Hiei et al., 1994). The control plants and independent transgenic lines were grown in a paddy field at Jinghong (southern of Yunnan province, $22^{\circ} 01^{\prime} \mathrm{N} / 100^{\circ} 49^{\prime} \mathrm{E}$ ) under natural conditions. Field management was performed with standard procedures to prevent yield loss during the growth period. Phenotypic data were collected at the maturing stage.

\section{Off-Target Detection}

The potential off-target sites were predicted on the website http://crispr.hzau.edu.cn/CRISPR/. DNA fragments containing the off-target sites were amplified by PCR using KOD DNA polymerase (Aidlab, Beijing, China). Then, the PCR products were sequenced and analyzed for detecting the off-target events. All the primers are in Additional file 1: Table S1.

\section{Trait Measurement}

All the transgenic and control plants were grown in the field under natural conditions with a plot size $100 \times 180$ $\mathrm{cm}^{2}$. The plant height, panicle length, panicle number, panicle branch number and grain number per panicle were obtained at the mature stage. The grain length, width, and thickness were measured by an electronic digital display Vernier caliper and fully filled grains were used for determined the 1000-grain weight. For grain quality, chalky percentage and degree of chalkiness were measured as the previous described (Hu et al. 2015b).

\section{Subcellular Location of the OsSPL4}

To verify the subcellular localization of OsSPL4 protein, the complete coding sequence without stop codon was amplified and cloned into the HBT-GFP vector driven by the cauliflower mosaic virus $35 \mathrm{~S}$ promoter. The 35S:OsSPL4-GFP fusion vector and nuclear localization vector (NLS-mCherry) were co-conducted into protoplast via PEG4000 inducing. After incubated in $28^{\circ} \mathrm{C}$ for $16 \mathrm{~h}-20 \mathrm{~h}$, the green and red fluorescence were observed with a confocal laser scanning microscope.

\section{Scanning Electron Microscopy}

Fresh young panicles were harvested and immediately fixed in $2.5 \%$ glutaraldehyde (in $25 \mathrm{mM}$ phosphate buffer, $\mathrm{pH}$ 7.0) overnight and then dehydrated through an ethanol series. Then, the samples were dried to critical point and sputter-coated samples were observed using a scanning electron microscopy (SEM) (Hitachi S-3400 N, Japan) as described previously (Gao et al. 2015).

\section{Dual Luciferase (LUC) Analysis in Tobacco Leaves}

The reporter gene constructs (GAL4-UAS) and effector constructs (SPL4, SPL4-d3, SPL4-d15) were performed as previously described (Lyu et al., 2020). Briefly, the GAL4-UAS was cloned and inserted into the pGreenII 0800-LUC vector as the reporter. The full-length CDSs of OsSPL4, OsSPL4-d3, OsSPL4-d15 were inserted into the pGreenII 62-SK vector as effectors. The empty vector pGreenII 62-SK was used as a negative control. The Agrobacterium strain containing both the reporter pGreenII0800-LUC and the helper pSoup-P19 was used either alone or mixed with the Agrobacterium strain containing the effector plasmids. Overnight cultures of Agrobacterium were collected by centrifugation resuspended and infiltrated. After three days, $N$. benthamiana leaf samples were collected for the Dual-LUC assay using commercial Dual-LUC reaction (DLR) reagents (Promega, USA) according to the manufacturer's protocol. The ratio of LUC to REN activities was calculated as an indication of the final transcriptional activity. Three biological repeats were measured for each sample.

\section{RNA Ligase-Mediated 5'-RACE}

Total RNA from rice young panicles was ligated directly to the 5'RACE adapter using the 5'-Full RACE Kit (Takara, Japan) according to the manufacturer's protocol as described (Yi et al. 2013). The first and second rounds of PCRs were performed with the OsSPL4-specific primers GSP1 and GSP2 (Additional file 1: Table S1). After PCR amplification, the PCR products were gelpurified and cloned into pMD18-T vector (Takara) for sequencing.

RNA-Sequencing and Co-expression Network Construction Total RNA was extracted from young panicles (P2 P4) and flag leaves of the control plants and spl4- $d 3$ transgenic lines. Libraries were constructed and sequenced using an Illumina HiSeq 4000 platform with three biological replicates. After filtering, the clean reads were performed using the TopHat and Cufflinks package (version 
2.2.1) (Trapnell et al. 2012). And DEGs were identified using DESeq 2 with false discovery rate $(F D R)<0.05$ (Love et al. 2014). Gene ontology (GO) and KEGG enrichment analysis were performed using AgriGO and KOBAS3.0 (http://kobas.cbi.pku.edu.cn/) with $P<0.05$, respectively (Du et al. 2010; Xie et al. 2011). The heatmaps of differential expression patterns were performed by MeV4.7 (Saeed et al., 2003). Gene regulatory interactions and gene networks were download from RiceNet v2 (https:// www.inetbio.org/ricenet/) (Lee et al. 2015). Then, coexpression networks were visualized by Cytoscape 3.6.1 (Shannon et al. 2003). All the clean reads were deposited in the National Center for Biotechnology Information (NCBI) under the accessions: PRJNA773224.

\section{Quantitative Real Time RT-PCR}

Total RNA was extracted using TRIzol reagent (Invitrogen) from leaves, young panicles or other tissues in the transgenic plants and control plants. RNase-free DNase was used to degrade contaminated DNA from total RNA at $37{ }^{\circ} \mathrm{C}$ for $30 \mathrm{~min}$. For mRNA reverse transcription (RT-PCR), the first strand cDNA was synthesized from $5 \mu \mathrm{g}$ total RNA with Oligo(dT) primer according to the manufacturer's instructions (Thermofisher Scientific). For miRNA RT-PCR, $2 \mu \mathrm{g}$ of total RNA was reverse transcribed using miRNA-specific stem-loop primers (Varkonyi-Gasic et al. 2007). The reactions were incubated for $30 \mathrm{~min}$ at $16{ }^{\circ} \mathrm{C}$, followed by 60 cycles of $30{ }^{\circ} \mathrm{C}$ for $30 \mathrm{~s}$, $42{ }^{\circ} \mathrm{C}$ for $30 \mathrm{~s}$ and $50{ }^{\circ} \mathrm{C}$ for $1 \mathrm{~s}$, then terminated by heating at $70{ }^{\circ} \mathrm{C}$ for $5 \mathrm{~min}$. All the primers are listed in Additional file 1: Table S1.

The qRT-PCRs were carried out with a StepOnePlus Real-Time PCR System (Applied Biosystems). The $\beta$-actin gene and U6 snRNA were used as an internal control for mRNA and miRNA qRT-PCR analysis, respectively. All cDNAs were diluted 5 times and $1 \mu$ diluted product was mixed with $5 \mu$ lof $2 \times$ SYBR Green mix (Roche) and $0.25 \mu \mathrm{M}$ primers in a $10 \mu \mathrm{l}$ volume reaction system, which were incubated $10 \mathrm{~min}$ at $95{ }^{\circ} \mathrm{C}$, followed by 40 cycles of $1 \mathrm{~min}$ at $60^{\circ} \mathrm{C}$. Three replicates were carried out for each sample, and the melting curve was performed to avoid nonspecific amplification. The relative expression levels were calculated using $\triangle \triangle \mathrm{Ct}$ method. All primers used in the present study are listed in Additional file 1: Table S1.

\section{Gene Structure and Protein Structure Analysis}

The exon and intron structures of individual OsSPL genes were illustrated using the GSDS website (Gene Structure Display Server, http://gsds.cbi.pku.edu.cn/) by aligning the genomic DNA sequences and the corresponding transcript sequences from the RAP-DB or RGAP databases (Hu et al. 2015a). Homology modelling of OsSPL4 protein was carried out using Swiss-Pdb View 4.1.0 with the Arabidopsis SPL4 (PDB: 1ul4) as the template. Protein structure and surface were visualized using PyMol software (https://pymol.org/).

\section{Phylogenetic Analysis of the SPL Genes}

Multiple sequence alignments of the 19 OsSPL gene and protein sequences were conducted using ClustalX software, and phylogenetic trees of the genes and proteins in rice were constructed with MEGA 7.0 software, respectively (Kumar et al. 2016). The phylogenetic trees were constructed using a neighbor-joining (NJ) method with bootstrap analysis of 1000 replicates.

\section{Nucleotide Diversity and Haplotype Network Analysis}

Nucleotide diversity $(\pi)$ was calculated to investigate the domestication of OsSPL4 in rice using 10 wild and 3010 cultivated rice accessions (Zheng et al. 2015). Fixation statistics $\left(F_{\mathrm{ST}}\right)$ among different subpopulations was calculated with sliding windows of $20 \mathrm{~kb}$ across the chromosome 2 based on the genetic variations of 529 rice accessions (Zhao et al. 2015). Haplotype network was constructed using an $\mathrm{R}$ package pegas with some modifications, and only haplotypes detected in more than 10 rice accessions were used.

\section{Results \\ Mutation of OsSPL4 Has Pleiotropic Effects on Rice Development}

To get a better insight into the role of SBP domain transcription factors in plant development, a CRISPR/Cas9 system was used to generate mutants of the SBP-box genes. In the present study, we identified the mutation lines of OSSPL4 to affect panicle development and grain size (Fig. 1). The CRISPR/Cas9 constructs targeted the SBP domain in the first exon of OsSPL4 was generated, which yielded 3 bp (spl4-d3) and 15 bp (spl4-d15) deletion, respectively (Fig. 1A, B). The potential off-target events in the mutation lines were also examined and the results showed that two of the six sites experience offtarget events $(10 \% \sim 20 \%)$ (Table 1). Interestingly, all these OsSPL4 mutation lines showed the similar phenotypes and might influence the plant height in the field experiments (Fig. 1C-I; Additional file 1: Fig. S1). These mutation lines exhibited long flag leaf, increased panicle length with more spikelets per panicle, and even increased the grain size (Fig. 1C-F). However, tiller number per plant of the mutation lines in the field experiments showed no significant difference (Additional file 1: Fig. S1). In addition, for grain quality, the chalky percentage and degree of chalkiness were increased in the mutation lines (Additional file 1: Table S2). Therefore, the results suggested 


\section{A OsSPL4}

Control Nip GGTGGGGAGGTGAGGTGCCAGGTGGAAGGGTGCGGGGTGGAGCT sp/4-d3 GGTGGGGAGGTGAGGTGCCA---GGAAGGGTGCGGGGTGGAGCT spl4-d15 GGTGGGGAGGTGAGGTG-_-_-_-_CGGGTGAGCT

B sp/4-d3
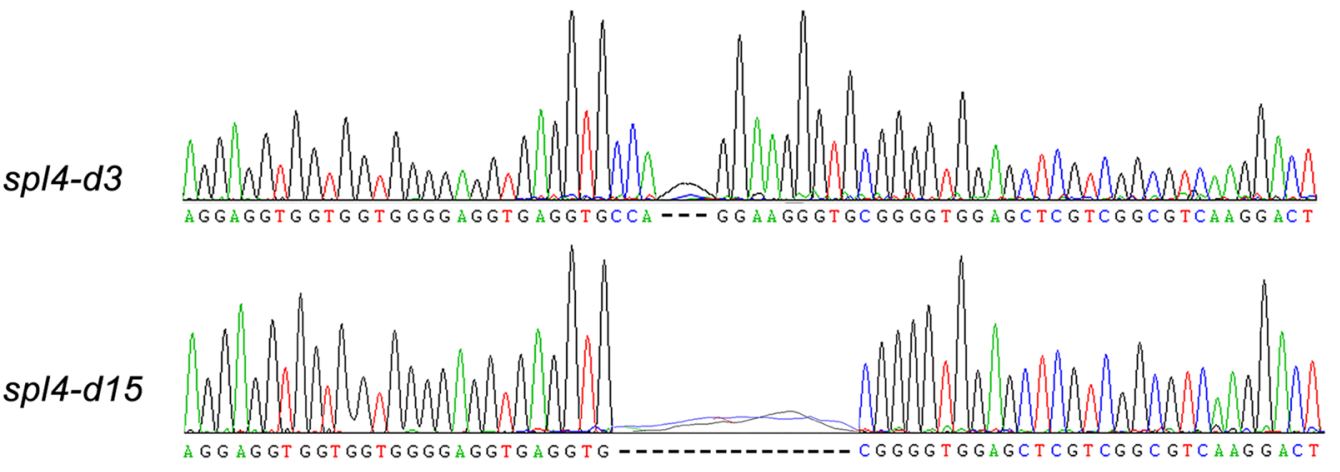

C

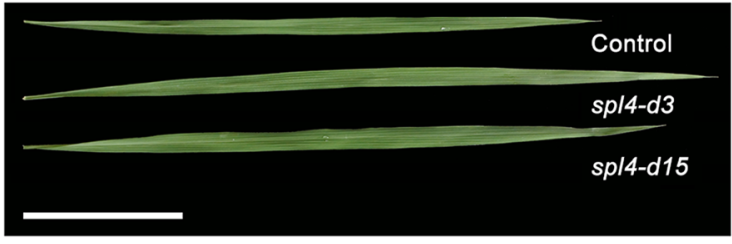

E
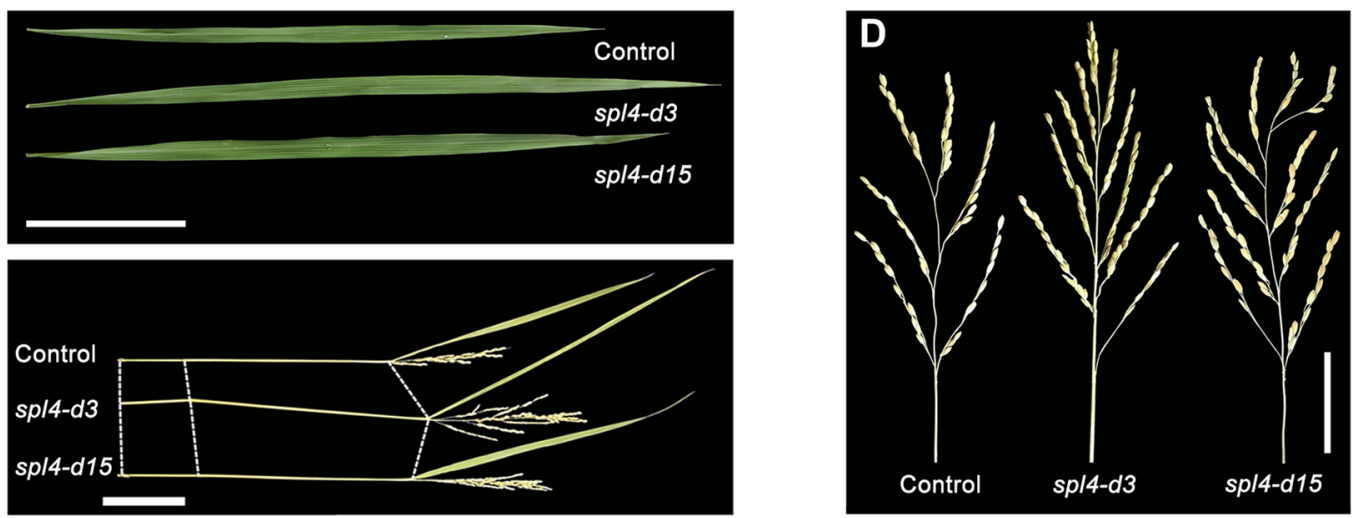

$\mathbf{F}$
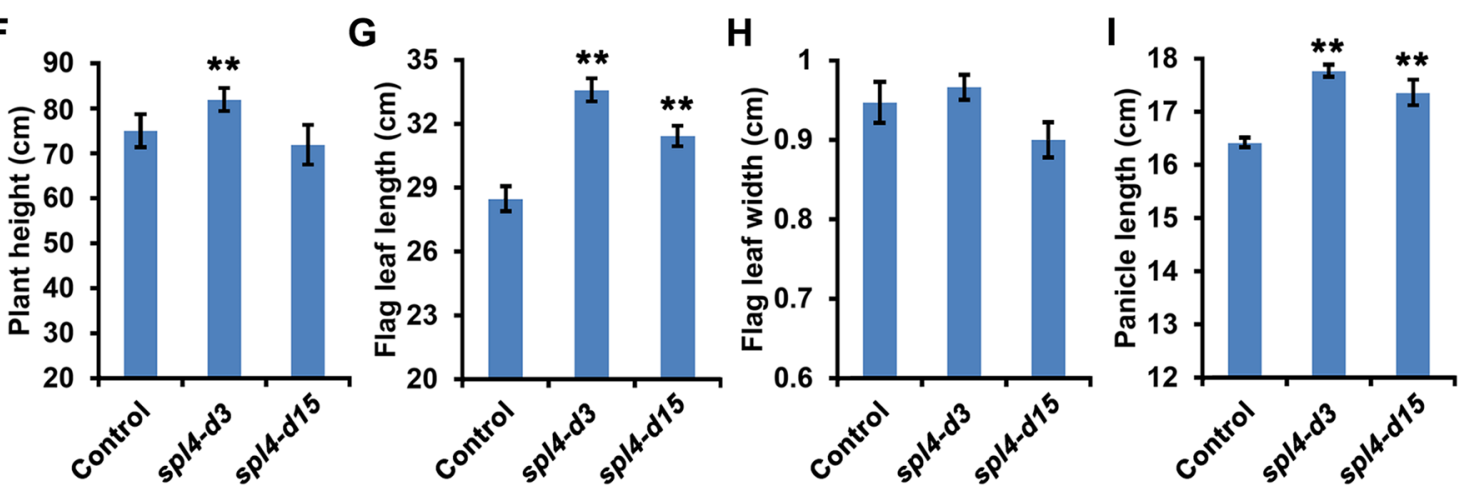

Fig. 1 Pleiotropic effects of the mutants of OSSPL4 in Nipponbare background. A Gene structure of OsSPL4 and the sequence alignment between the mutants in T3 generation and control plants. Nip, Nipponbare. The deleted amino acid residues in the mutants are presented by dashes. The protospacer adjacent motif (PAM) are underlined in red. Two types of mutation events (sp/4- $d 3$ and sp/4-d15) were generated by CRISPR/Cas 9 in Nipponbare rice. B The two different mutation events were confirmed by Sanger sequencing. C-H Comparisons of several important traits among spl4-d3, sp/4-d15 lines and control plants. C Flag leaf, scale bar $=10 \mathrm{~cm}$. D Panicle, scale bar $=5 \mathrm{~cm}$. E Stem, scale bar $=10 \mathrm{~cm}$. F Plant height. G Flag leaf length. $\mathbf{H}$ Flag leaf width. I Panicle length. $P$ values were determined by Student's $t$ test. ${ }^{*} P<0.05,{ }^{* *} P<0.01$ 
Table 1 The putative off-target event in the ossp/4 mutant lines

\begin{tabular}{lllllcc}
\hline Target site & Putative off-target site & Sequence of putative off-target site & Region & No. of plants & $\begin{array}{l}\text { No. of plants } \\
\text { with mutations }\end{array}$ & $\begin{array}{l}\text { Mutation } \\
\text { rate (\%) }\end{array}$ \\
\hline OsSPL4-OFF1 & Chr7:19100187-19100209 & AGGTGCCAGGTGGAGAGGTGCGG & CDS & 10 & 2 & $20 \%$ \\
OsSPL4-OFF2 & Chr8:25274878-25274900 & CGGTGCCAGGTGGAGGGGTGCGG & CDS & 10 & 1 & $10 \%$ \\
OsSPL4-OFF3 & Chr9:18918476-18918498 & AGGTGCCAGGTGGAGGGTTGCGG & CDS & 10 & 0 & 0 \\
OsSPL4-OFF4 & Chr8:167437-167459 & TGGTGCTAGGAGGAAGGGTTTGG & Intergenic & 10 & 0 & 0 \\
OsSPL4-OFF5 & Chr9:16055179-16055201 & AGAGGCCTGGTGGAAAGGTGAGG & Intron & 10 & 0 & 0 \\
OsSPL4-OFF6 & Chr1:18039404-18039426 & AAGCGCCATGTGGAGGGGTGTGG & Intergenic & 10 & 0 & 0 \\
\hline
\end{tabular}

The bold font represents the PAM motif (NGG), the italics font indicates the mismatch bases

that OsSPL4 plays an important role in panicle and grain development.

\section{Mutation of OSSPL4 Increases Grain Weight}

The spikelet hull of OsSPL4 mutation transgenic plants was apparently larger than that of control plants both in length and width (Fig. 2A). The grain length of spl4-d3 and spl4-d15 lines was significantly longer than that of the control plants, and grain width were also increased in spl4- $d 3$ and spl4- $d 15$ mutation lines, while the grain thickness were decreased (Fig. 2B-D). And the grain bulk density in spl4- $d 3$ and spl4- $d 15$ lines were significantly decreased (Fig. 2E). Thus, the 1000-grain weight was increased in spl4-d3 and spl4-d15 lines, compared with the control plants. Furthermore, the grain yield per plant was significantly increased by $11.44 \%$, and the yield per plot was also increased by $5.35 \%$ in the spl4- $d 3$ lines, but the yield in spl4-d15 lines were not significantly increased (Fig. 2F, G and Additional file 1: Table S2).

\section{Overexpression of OsSPL4 Decreases Grain Weight}

To further confirm the role of OSSPL4 in controlling grain size, we generated an overexpression construct driven by the Ubi promoter and introduced it into Nipponbare rice. Compared with the control plants, the grain size and grain weight in overexpression lines were decreased (Fig. 3). One of the transgenic lines (OE-2) showed apparently decrease in grain length and grain width as well as 1000-grain weight, while the grain thickness were increased (Fig. 3A-E). And the decreased grain size was further confirmed to be the consequence of highly expression level of OsSPL4 with 6.0 and 13.5 fold increase in OE-1 and OE-2 lines, respectively (Fig. 3F). These results further suggested that OsSPL4 regulated grain size in rice.

\section{Mutation of OsSPL4 Increases Grain Size by Promoting Cell Division}

Compared with the spl4-d15 lines, the 3 bp mutation of OsSPL4 ( spl4-d3) transgenic plants exhibited better field phenotypes, which could be an excellent allele for regulating grain size in rice (Figs. 1, 2 and Additional file 1: Table S2). Therefore, we used the spl4- $d 3$ lines for further functional studies. Scanning electron microscopy of the outer glume revealed that spl4- $d 3$ lines exhibited a significantly enlarged cell volume than that of control plants (Fig. 4A, B). And the cell length in longitudinal direction was larger in spl4- $d 3$ lines with an increase in epidermal cell numbers per unit area (6.85\%) (Fig. 4C, D). Furthermore, young panicles of the spl4- $d 3$ transgenic lines and control plants were analyzed using RNA-seq. KEGG pathway analysis revealed that many differentially expressed genes (DEGs) were involved in cell cycle pathway, MAPK signaling pathway and steroid biosynthesis (Fig. 4E). Many genes in the cell cycle pathway were highly expressed in the spl4- $d 3$ transgenic plants (Fig. $4 \mathrm{~F}$ and Additional file 1: Table S3). The expression levels of 10 cell-cycle related genes were validated by qRTPCR, and many of these genes, including CYCA3-1 and CYCD2-1 were significantly up-regulated (Fig. 4G). These results suggested that a higher expression of the cell-cycle genes probably promoted cell proliferation, contributing to affect grain size in spl4- $d 3$ mutation transgenic plants, which might potentially be useful for the improvement in rice yield.

\section{Mutation of OsSPL4 Promotes Panicle Branching}

In our study, spl4- $d 3$ transgenic plants possessed more spikelets per panicle and effective grains per panicle than those of control plants and spl4-d15 lines, resulting in more grain number and significantly increase in rice yield (Fig. 5A-D, Additional file 1: Table S2). The seed setting rate was also slightly increased in the spl4$d 3$ lines (Fig. 5E). The increased spikelets of spl4-d3 lines were attributed to the increase of both primary branches and secondary branches (Fig. 5B, F, G). Scanning electron microscopy also showed that much more spikelets in the spl4- $d 3$ lines than those of control plants (Fig. 5C). Using RNA-seq, many floral identity genes in classes $\mathrm{A}, \mathrm{B}, \mathrm{C}, \mathrm{E}$, and AGL6 of the ABCDE 
A

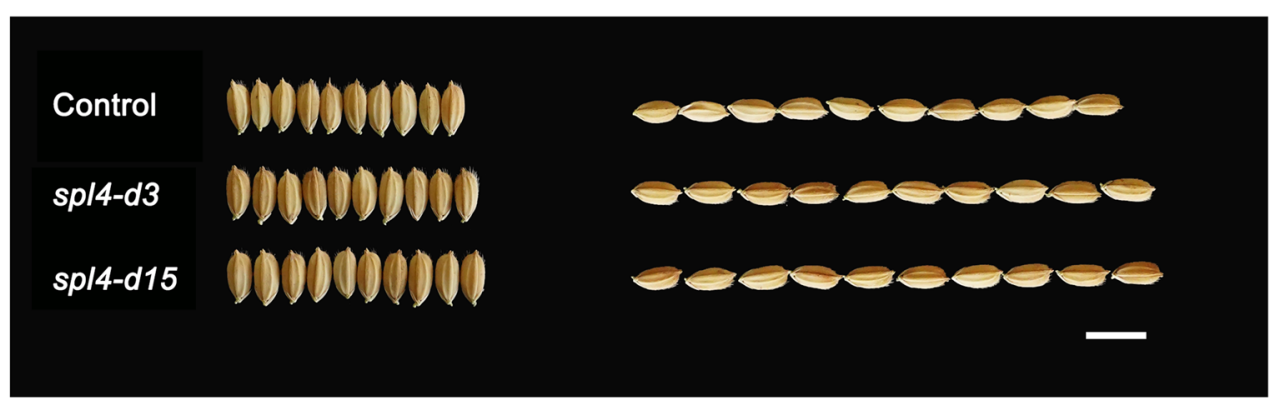

B

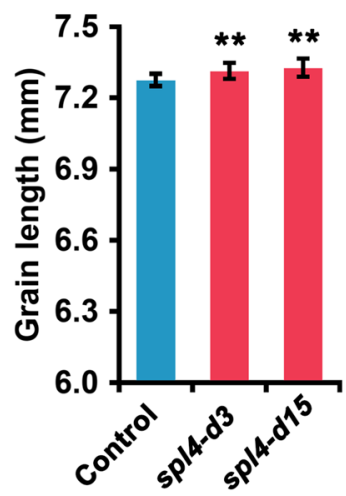

E

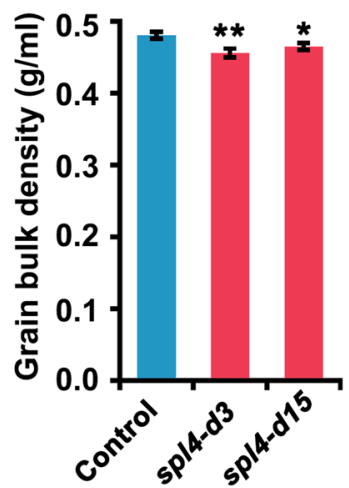

C

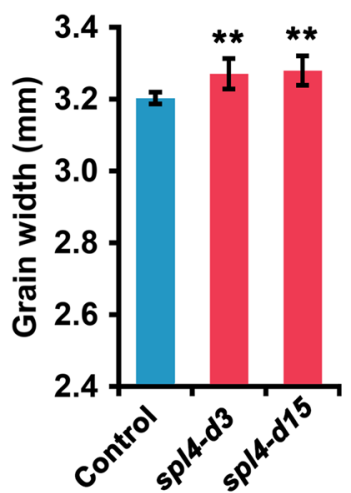

$\mathbf{F}$

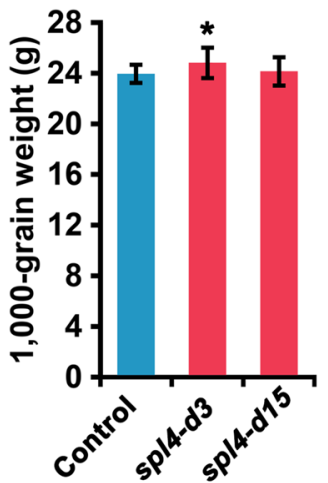

D

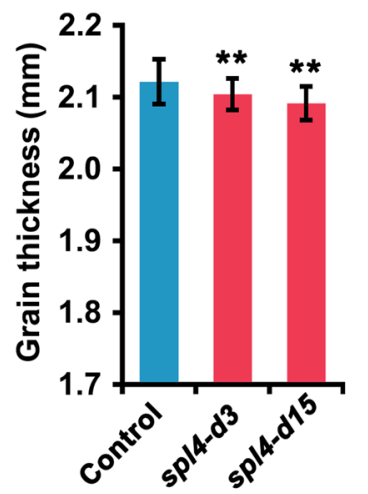

G

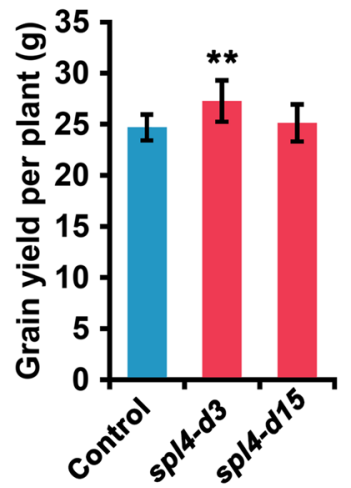

Fig. 2 Grain phenotypes of the OsSPL4 mutation transgenic lines and control plants. A Grain shapes of sp/4- $d 3$, sp/4- $d 15$ and control plants, scale bar $=1 \mathrm{~cm}$. B-G Comparisons of grain size and the yield between sp/4-d3, sp/4-d15 and control plants. B Grain length. C Grain width. D Grain thickness. E Grain bulk density. F 1000-grain weight. G Grain yield per plant. Significant difference was determined by Student's $t$ test, ${ }^{*} P<$ $0.05,{ }^{* *} P<0.01$

model were observed to be up-regulated in the young panicles (Fig. 5H, Additional file 1: Table S3). Further qRT-PCRs validated the highly expression levels of some MADS genes in the spl4-d3 lines (Fig. 5I). The results indicated that OsSPL4 regulated some of the floral genes in the mutation lines, which may eventually result in more panicle branches and grains per panicle. The number of panicle branches determines the number of grains, which is a major factor in affecting grain yield in rice.

\section{Identification of Genes Regulated by OsSPL4}

To further investigate the molecular mechanism of OsSPL4 on panicle and flag leaf development as well as affecting grain size, RNA-seq analysis was carried out using the young panicles at $\mathrm{P} 2 \sim \mathrm{P} 4$ stages and flag leaves from the control and spl4- $d 3$ transgenic plants, respectively. A total of 3,816 and 238 DEGs were identified in the young panicles and flag leaves, respectively (Additional file 1: Fig. S2A). GO analysis showed that "metabolic process", "macromolecular complex assembly" and "oxidation reduction" were significantly enriched in the 
A
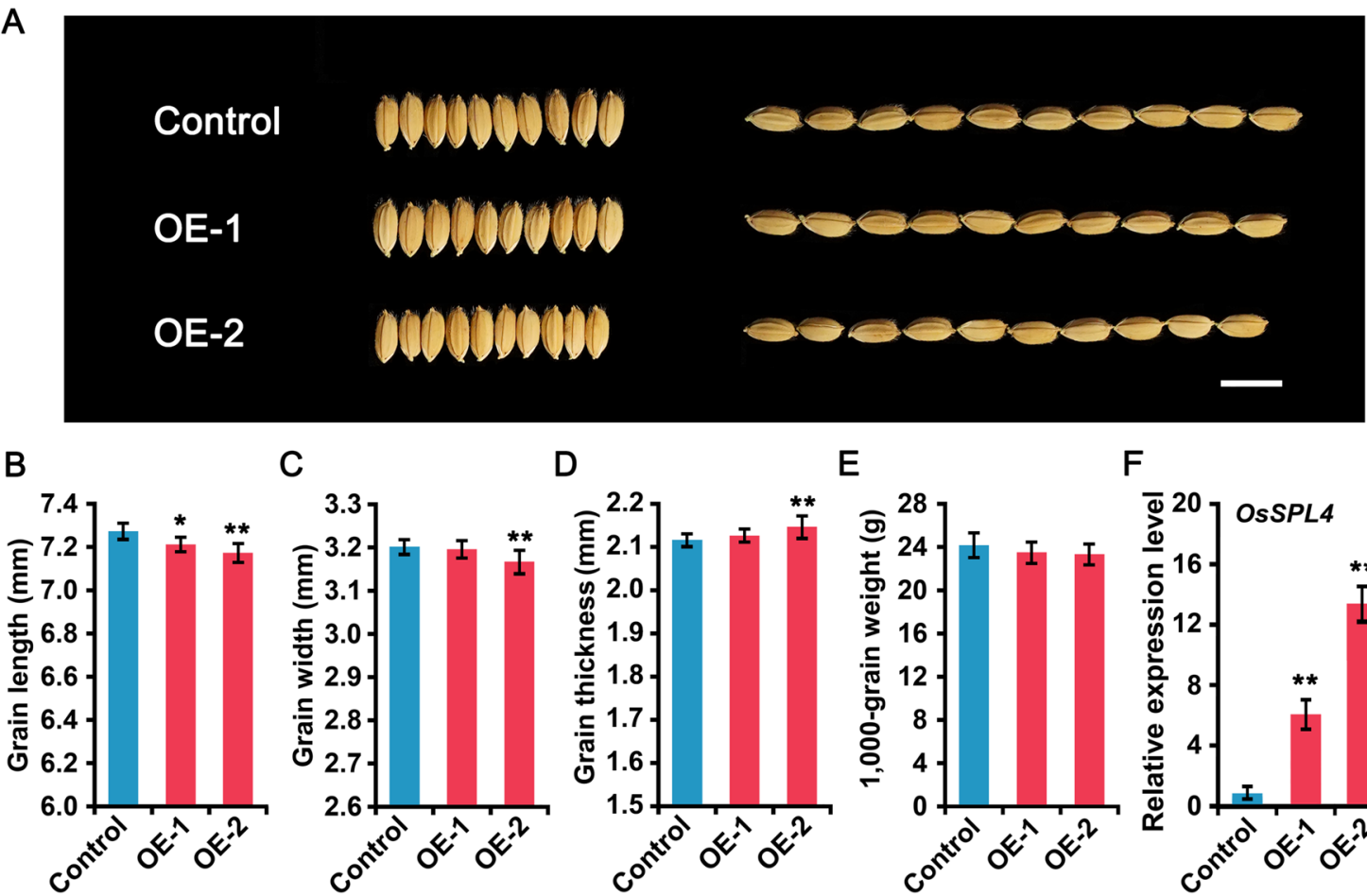

C
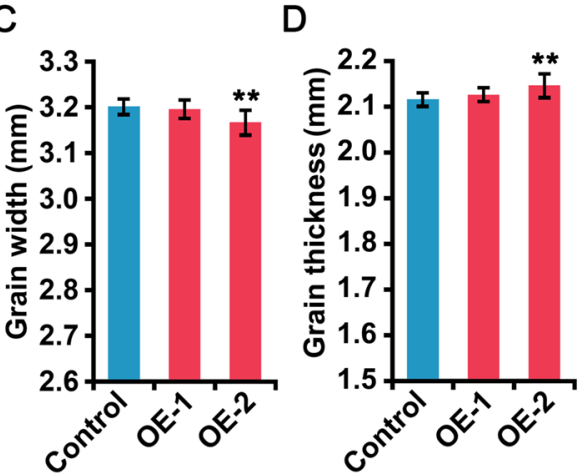

E

$\mathrm{F}$
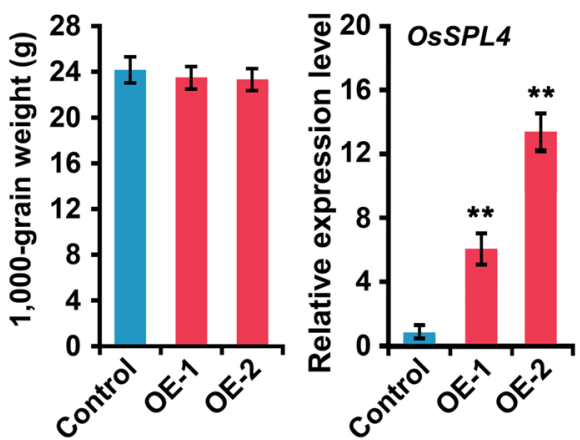

Fig. 3 Overexpression of OsSPL4 decreases rice grain size and weight. A Grain comparisons of the control and OsSPL4 overexpression plants, scale $\mathrm{bar}=10 \mathrm{~mm}$. B-E Comparisons of the grain size and expression patterns in the control and OsSPL4 overexpression plants. B Grain length. C Grain width. D Grain thickness. E 1000-grain weight. F The expression levels of OsSPL4. Significant difference was determined by Student's $t$ test, ${ }^{*} P<$ $0.05, * * P<0.01$

tissues between control and spl4- $d 3$ lines (Additional file 1: Fig. S2B,C). KEGG pathway analysis revealed that many DEGs participated in carbon metabolism, MAPK signaling, amino acid metabolism and ubiquitin mediated proteolysis (Fig. 4E; Additional file 1: Table S4). Particularly, we detected 16 and 7 DEGs to be involved in cell cycle and steroid biosynthesis, respectively. And a total of 21 MADS-box genes and 18 cell-cycle genes were up-regulated in the spl4-d3 lines (Fig. 5H, Additional file 1: Table S3). Quantitative RT-PCR also validated the expression patterns of the four OsMADS genes and 10 cell-cycle genes (Fig. 4G, Fig. 5I).

To date, many rice yield-related genes, such as $R F L$, OsGIF1, RGG2, DEP2, LAC, TGW3, and GL7/SLG7 have been reported to play essential roles in controlling the panicle architecture and grain size (Hu et al. 2020; Miao et al. 2019). In our study, we found that many of these yield-related genes had been differentially expressed in the spl4- $d 3$ lines (Additional file 1: Table S5). Especially, several genes including GL3.3/TGW3 (OsSK41), LAC, and $R G G 2$ which negatively regulated rice grain size, were significantly down-regulated in the spl4- $d 3$ lines. By contrast, other genes of OsGIF1, RGB1, and GL7/SLG7 were highly expressed in the spl4-d3 lines (Additional file 1: Table S5). In addition, some of the BR signaling genes, including $B I M 2$ and $O s B A K 1$ were also slightly upregulated in the spl4-d3 lines (Additional file 1: Table S4). Co-expression network revealed that the regulation network of OSSPL4 interacted with the yield-related genes, indicating the possible pathways of OsSPL4 contributed to rice yield (Additional file 1: Fig. S3).

\section{The Role of SBP Domain in OsSPL4 Protein}

There are 19 OSSPL genes in the rice genome, which have 3 to 11 exons (Additional file 1: Fig. S4A). Sequence analysis of the 19 OsSPL genes showed that OsSPL4 has synteny with OsSPL11 on chromosome 6 for segmental duplication in rice (Additional file 1: Fig. S4A). However, the last exon of OsSPL4 is degenerated to the $3^{\prime}$-UTR which retained the OsmiR156 target site. Phylogenetic analysis of the OsSPL proteins revealed that the rice OsSPLs could be divided into 6 subgroups based on their different domains (Additional file 1: Fig. S4B). All of the rice OsSPL proteins have SBP domain. Expectedly, the syntenic OsSPL proteins were clustered in the same group and had the similar protein structures (Additional file 1: Fig. S4B). 
A

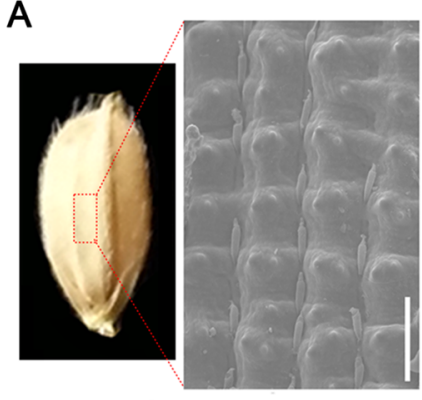

Control
B

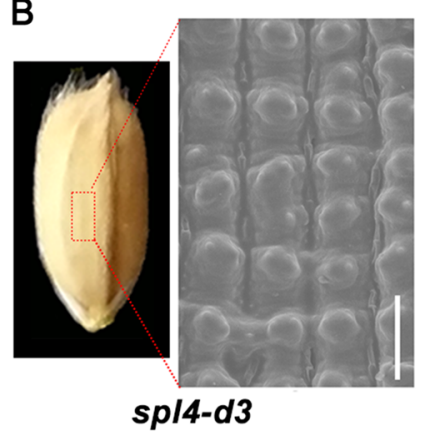

spl4-d3

E

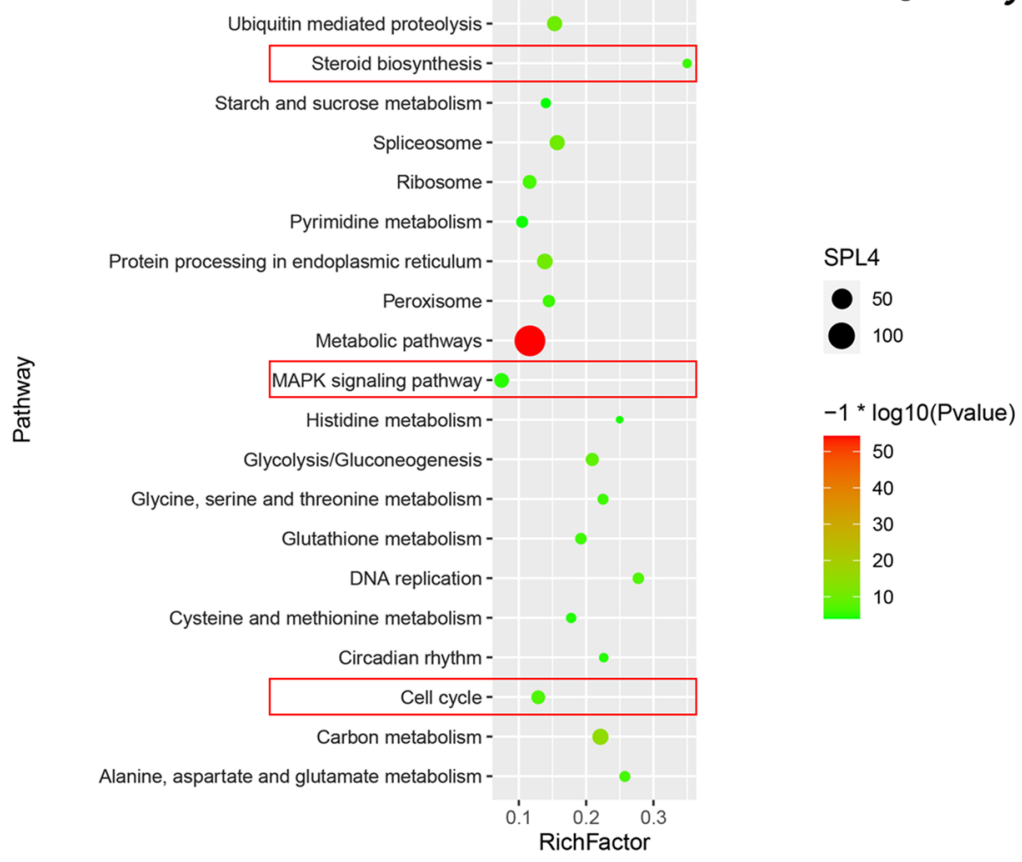

C

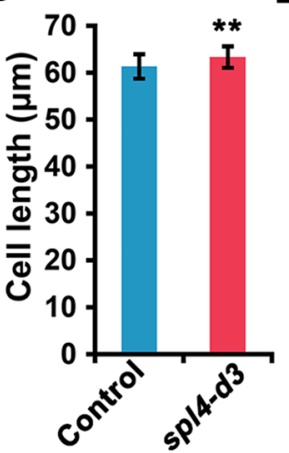

$F$
D

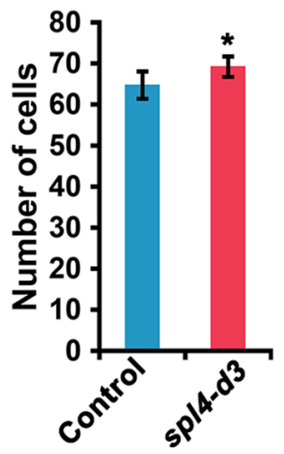

$\begin{array}{lll} & & \\ 0 & 1 & 2\end{array}$

CYCA3-1

CYCB1-2

CYCB2-2

CYCD2-1

CYCD3-1

CYCD5-3

CDKA-2

CDKB-1

CDKB-2

CDKG-2

CKL3

DEL1

MCM2

MCM3

MCM4

MCM5

G

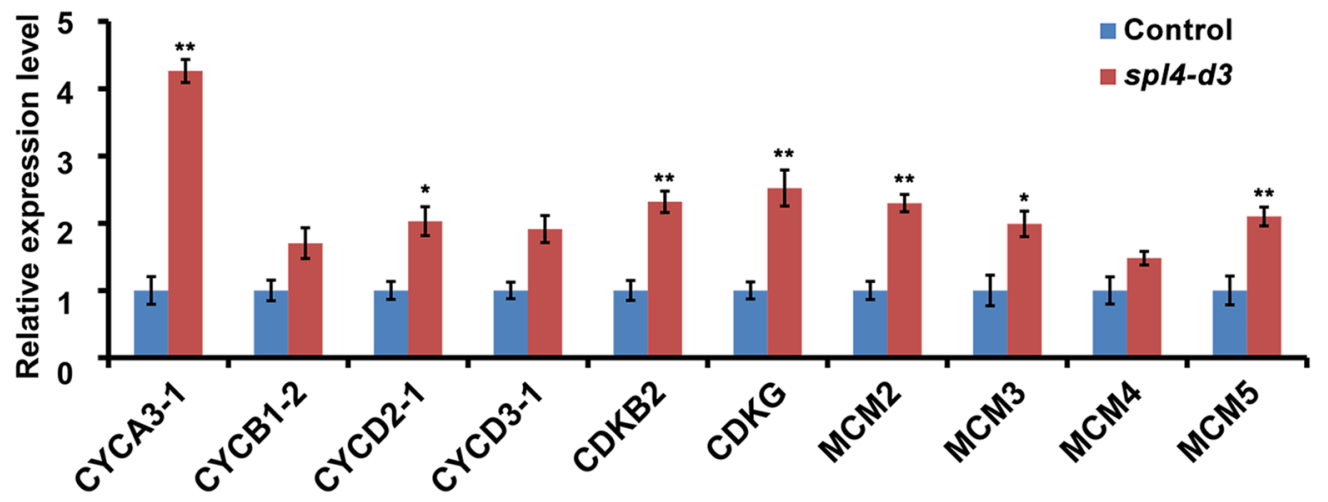

Fig. 4 OSSPL4 regulates spikelet hull development by affecting cell proliferation A, B Scanning electron microscope images of the outer glume (middle), A Control plants; B spl4- $d 3$ lines, scale bar $=100 \mu \mathrm{m}$. C, D Comparisons of cell length and cell number in the longitudinal direction of the spikelet between the control and sp/4- $d 3$ plants ( $n=10$ spikelets). C Cell length. D Cell number. E KEGG pathway of the DEGs identified in young panicles of the control and sp/4-d3 transgenic plants. RichFactor is the ratio of DEG number and background gene number. $\mathbf{F}$ Heatmap showing the expression levels of genes related to floral organ identify in our RNA-seq between the spl4- $d 3$ lines and control plants. Fold change of the FPKM values (sp/4-d3/control) were used. G Comparisons of the expression levels of cell-cycle genes in young panicles of the control and sp/4-d3 plants using qRT-PCR. The data are presented as mean $\pm \mathrm{SD}(\mathrm{n}=3)$. Significant difference was determined by Student's $t$ test, ${ }^{*} P<0.05,{ }^{* *} P<0.01$ 
A

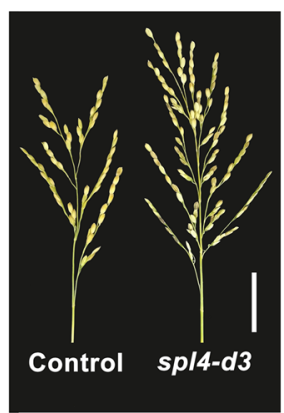

D

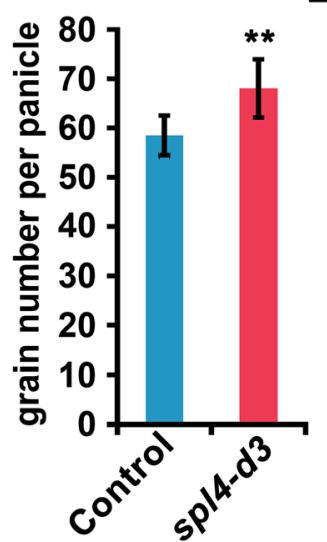

$\mathrm{H}$
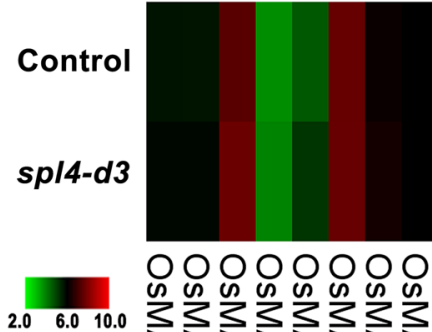

OOOOOOOO

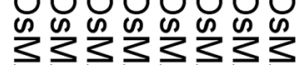

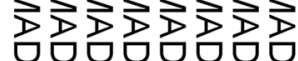

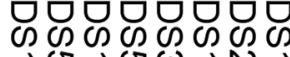
$\vec{v} \backsim \rightarrow \bigcup_{\infty} \omega \vec{\sigma} \vec{A}$

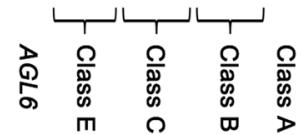

B

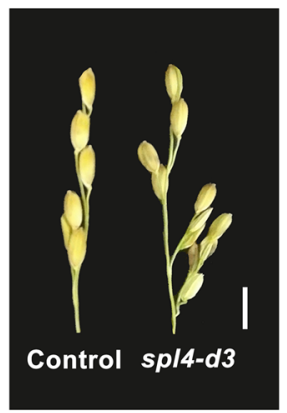

E

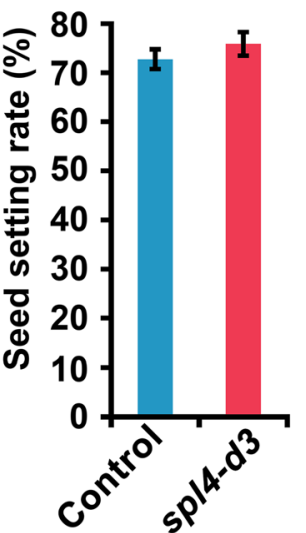

C

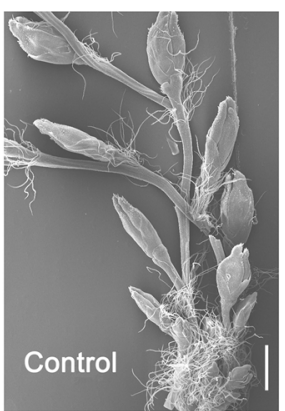

F

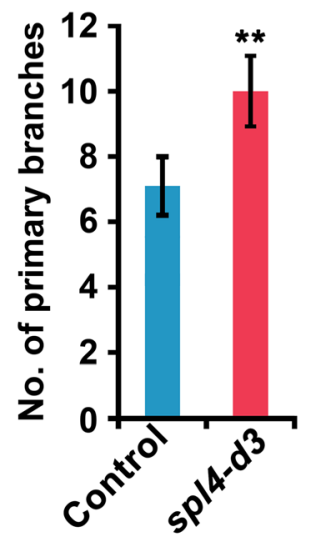

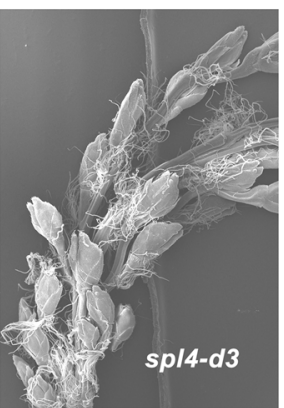

G

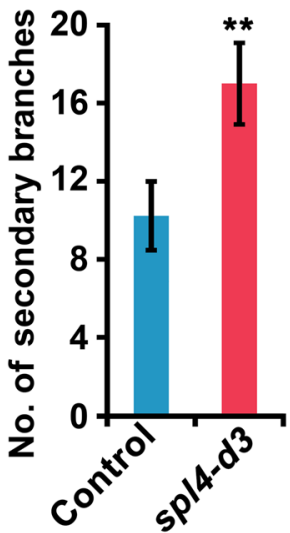

I

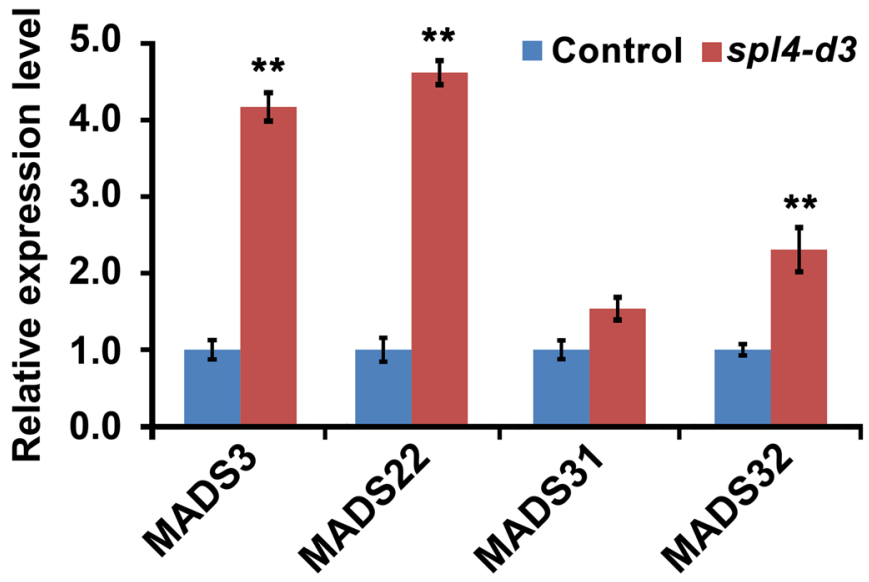

Fig. 5 Comparisons of the panicle morphologies of sp/4-d3 transgenic plants with control plants. A Panicle architecture, scale bar $=5 \mathrm{~cm}$. B Secondary branch, scale bar $=1 \mathrm{~cm}$. C Scanning electron micrographs of the young panicle, scale bar $=4 \mathrm{~mm}$. D Grain number per panicle. E Seed setting rate. $\mathbf{F}$ Number of primary branches. $\mathbf{G}$ Number of secondary branches. $\mathbf{H}$ Heatmap of the expression patterns of the floral identity genes. I qRT-PCRs validation of the expression levels of some flower development genes (MADS3, MADS22, MADS31 and MADS32) in sp/4-d3 transgenic plants. Data were normalized to those of the control plants. Values are means $\pm S D(n=3)$. Significant difference was determined by Student's $t$ test, ${ }^{*} P<0.05,{ }^{* *} P<0.01$

In plants, the SBP-DBD that consists of two subdomains has a single zinc-binding pocket for each one, which is likely to be stabilized through interactions of hydrophobic residues, such as Val, Phe and Tyr (Yamasaki et al. 2006). In our study, we found that OsSPL4 also contains the typical SBP domain with two nuclear location signals (NLSs) (Fig. 6A and Additional file 1: Fig. S5). And the mutations of spl4-d3 and spl4-d15 deleted one and five amino acids in the SBP domain, respectively (Additional file 1: Fig. S5). Particularly, one of the 
A

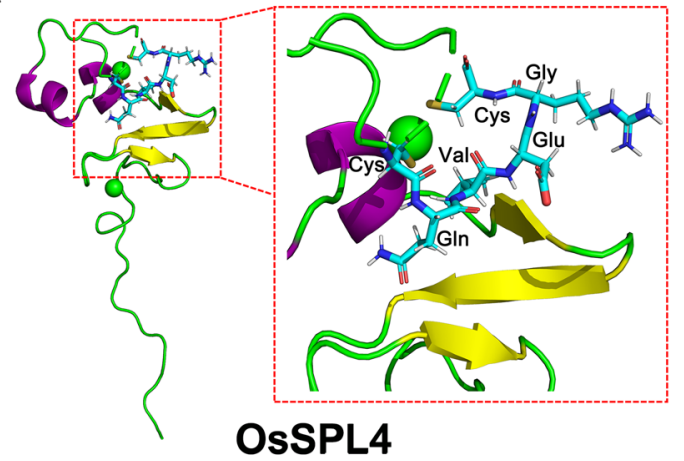

D

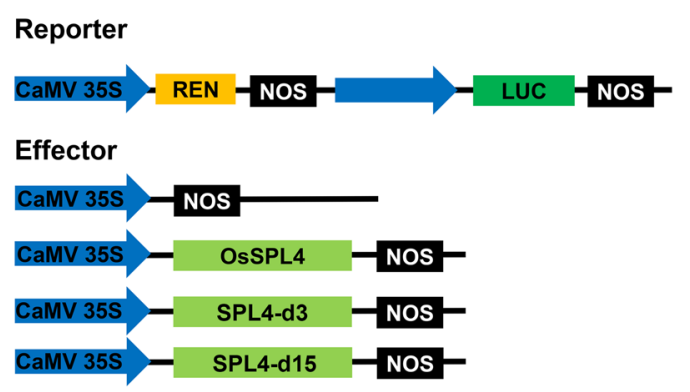

B

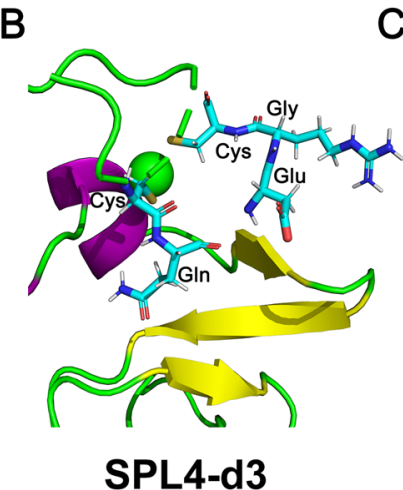

C

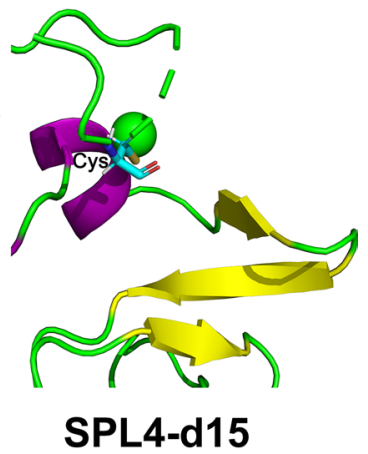

E

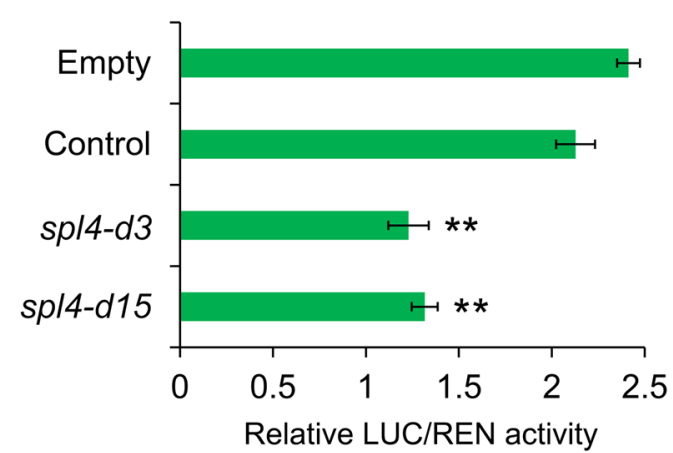

Fig. 6 Three-dimensional (3D) structures of the control and sp/4-d3 plants and expression patterns of OsSPL4. A Three-dimensional homology modeling of the OsSPL4 protein based on crystallography data from Arabidopsis AtSPL4 structure (PDB ID: 1ul4.pdb). B The 3D structure of OsSPL4 in sp/4-d3 lines. C The 3D structure of OsSPL4 in sp/4-d15 lines. D Schematic diagram of the reporter and effector constructs for dual-luciferase transient assays. E Effects of OsSPL4 in sp/4-d3 and sp/4-d15 on the transcriptional regulation in Nicotiana benthamiana. Values are means \pm SD $(n=3)$. Significant difference between control and sp/4-d3 or sp/4-d15 was determined by Student's $t$ test, ${ }^{*} P<0.05,{ }^{* *} P<0.01$

Cys residues which participates in the zinc binding was deleted in the spl4- $d 15$ mutation lines, resulting in not forming $\mathrm{C} 3 \mathrm{H}$ subdomain. The $3 \mathrm{D}$ structure analysis also revealed that the lack of Val residues could affect formation of the first zinc-binding pocket in spl4-d3 mutation of OsSPL4 (Fig. 6B). Whereas, the $15 \mathrm{bp}$ mutation of OsSPL4 devastate the binding pocket due to the deletion of Cys residue (Fig. 6C).

To investigate whether the two mutation (SPL4d3 and SPL4-d15) of OsSPL4 were loss of functions, we performed a dual-luciferase transient transcriptional activity assay in $N$. benthamiana leaves with OsSPL4 driven by the CaMV $35 \mathrm{~S}$ promoter as an effector and LUC as the reporter gene (Fig. 6D). DEP1 is an important regulator for panicle architecture and elevated expression of DEP1 contributed to increasing panicle length (Zhou et al., 2009; Lu et al., 2013). Previous studies have demonstrated that OsSPL genes could bind to the promoter of DEP1 (Lu et al., 2013; Yuan et al. 2019). In our study, the results showed that the extent of DEP1::LUC were significantly reduced by cotransformation with OSSPL4 in both SPL4-d3 and SPL4-d15 compared with the control plants (Fig. 6E), indicating the transcriptional activity of these two mutations of OsSPL4 were reducing. These results suggested that the two mutations of OSSPL4 (spl4$d 3$ and spl4-d15) in this study could affect the binding in promoter of regulated genes and then influence the regulation of downstream genes.

\section{Expression Pattern and Subcellular Location of OsSPL4}

To determine the tissue-specific expression levels of OsSPL4, qRT-PCR analysis in wild type plants revealed that the expression level of OSSPL4 was highly detected in the leaf and young panicle at P4-P6 stage during panicle development (Fig. 7A). But the expression level of OsSPL4 was weakly detected in roots, developing seeds of 5DAP and the 10DAP stages (Fig. 7A). Consistent with these expression patterns, mutation of OsSPL4 showed that flag leaf length, panicle length and the number of primary panicle branches as well as grain size were changed (Fig. 1). 


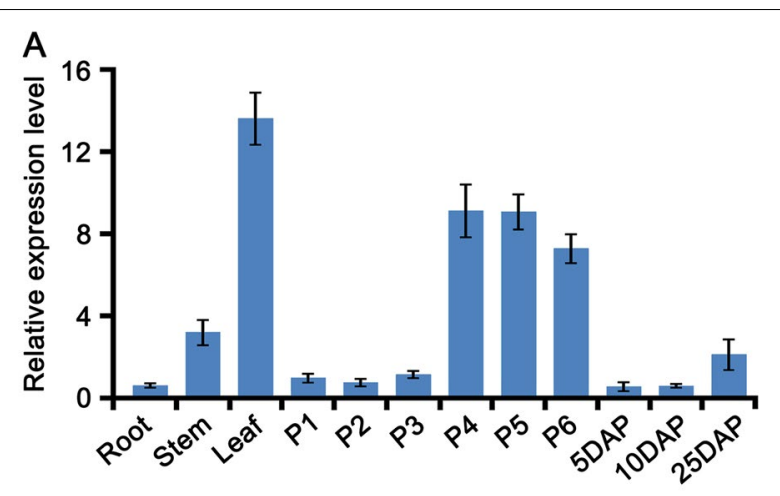

B
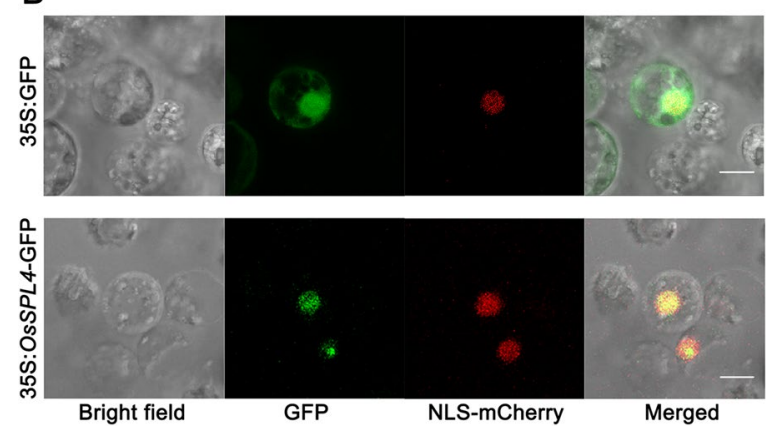

Fig. 7 The expression patterns and subcellular localization of OsSPL4. A The expression patterns of OSSPL4 in different tissues. P1-P6 indicates a plant with a panicle length of 0,3,5, 10, 15 and $22 \mathrm{~cm}$, respectively. DAP, days after pollination. B Subcellular localization of OsSPL4-GFP fusion protein. Scale bar $=10 \mathrm{~mm}$, applicable to all photos

To determine the subcellular location, the coding sequence of OsSPL4 was fused with GFP driven by the cauliflower mosaic virus $35 \mathrm{~S}$ promoter. The results showed that green fluorescence from OsSPL4-GFP fusion proteins was coincident in the same position with red fluorescence from the nucleus marker vector NLSmCherry, suggesting that OsSPL4 was located in the nucleus (Fig. 7B).

\section{OsSPL4 is One of the Osa-miR156 Targets}

Using psRNATarget, we predicted that OsSPL4 can be targeted by many members of OsmiR156 (Additional file 1: Fig. S6A). And the target site of OsmiR156 is located in the $3^{\prime}$-untranslated region (UTR) of OsSPL4. A 5'RLM-RACE (RNA ligase-mediated rapid amplification of cDNA ends) analysis showed that osa-miR156 could directly cleave OsSPL4 mRNA in vivo (Additional file 1: Fig. S6A). To further investigate whether the regulation of OsmiR156 on OsSPL4 affects the grain size, we generated the overexpression and STTM constructs, and then they were introduced into Nipponbare rice (Additional file 1: Fig. S6B). Moreover, qRT-PCRs revealed that four of the eight predicted targets were up-regulated in the
STTM156, while, only the OsSPL4 significantly downregulated in the OE-miR156 lines, indicating OSSPL4 was a target of osa-miR156 (Additional file 1: Fig. S6C). The OE-miR156 transgenic plants exhibited a larger grain size, which increased the grain length and width (Additional file 1: Fig. S7). Pearson correlation analysis showed that the expression levels of OsSPL4 in OE-miR156 lines were significantly negatively correlated with grain length $(P=0.0286, \mathrm{R}=0.6285)$ (Additional file 1: Fig. $\mathrm{S} 7 \mathrm{C})$. Moreover, one of the rice STTM156 line (STTM156-4) showed that the grain length and width were obviously decreased (Additional file 1: Fig. S8). Consistent with the above results, OsSPL4 transcript levels in the osa-miR156 overexpressing lines were lower than those in the control plants, whereas the expression level of OSSPL4 increased in the STTM156 transgenic plants (Additional file 1: Fig. S7B and S8B). And the Pearson correlation analysis also revealed that the expression levels of OsSPL4 in STTM156 lines were significantly negative correlated with grain length $(P=0.0024, \mathrm{R}=0.7862)$ and grain width $(P=0.0031, \mathrm{R}=0.7748)$ (Additional file 1 : Fig. S8E, F), respectively. These results suggested that OsSPL4 was one of the targets of osa-miR156, and down-regulated OsSPL4 by OsmiR156 might change the rice grain shape.

\section{Genetic Diversity of OsSPL4 Gene}

Compared with the wild rice SPLs, we observed that last exon of SPLs (OsSPL4 and OglaSPL4) in cultivated rice were truncated (Additional file 1: Fig. S9). The results indicated that OSSPL4 may be domesticated from wild rice. To further determine whether OsSPL4 has undergone artificial selection, we analyzed the genetic diversity in OSSPL4 and its flanking regions $(\sim 4 \mathrm{~kb})$ using 10 accessions of wild rice (5 accessions from O. rufipogon and 5 accessions from $O$. nivara) and 3010 cultivars of $O$. sativa (Fig. 8A). Compared with the nucleotide diversity ( $\pi)$ of wild rice O. rufipogon $(\pi=0.2308)$ and O. nivara $(\pi=0.2277)$, the nucleotide diversity in OsSPL4 genome sequences was decreased in indica and japonica cultivars $(\pi=0.1284$ and 0.0393 , respectively) (Fig. $8 B$ ). The results revealed that the nucleotide diversity of OsSPL4 gene in cultivars was lower than that in wild rice $(P<0.01)$, and that the diversity in japonica cultivars was lower than that in indica cultivars, suggesting OsSPL4 might have undergone artificial selection. Moreover, we examined OsSPL4 evolution by estimating the level of population difference $\left(F_{\mathrm{ST}}\right)$ on chromosome 2 between different subpopulations. Pairwise measurements of $F_{\mathrm{ST}}$ showed that $F_{\mathrm{ST}}$ levels were truly higher between indica and japonica in the OSSPL4 gene. However, markedly weaker differences were found between aus and indica in the OSSPL4 when compared with the mean $F_{\mathrm{ST}}$ over chromosome 2 as a whole (Fig. 8C). A minimum-spanning tree of 

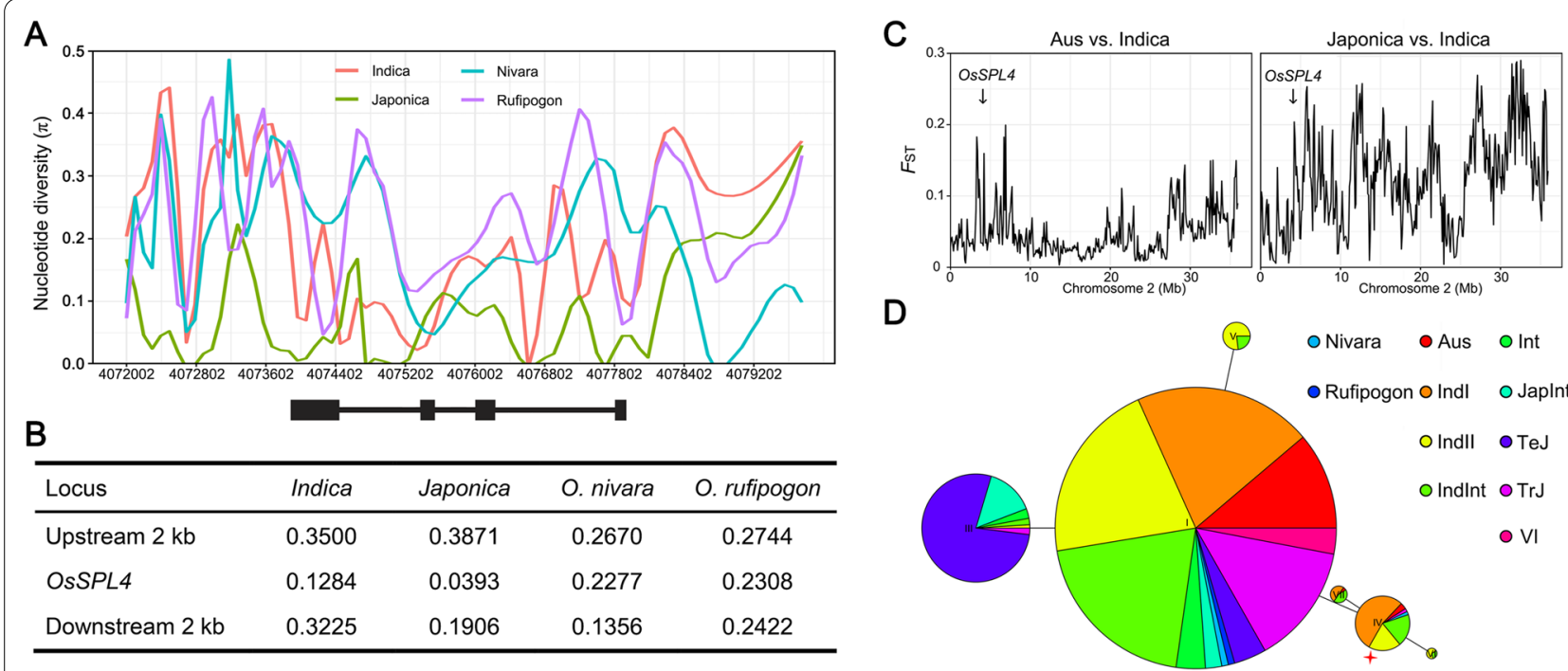

Fig. 8 The nucleotide diversity and haplotype network analysis of OSSPL4. A Nucleotide diversity ( $\pi$ ) analysis in OSSPL4 gene and its flanking region ( $4 \mathrm{~kb}$ ) based on the 10 wild and 3010 cultivated rice accessions. Nivara, O. nivara; Rufipogon, O. rufipogon. Y-axis indicates the $\pi$ value and $x$-axis shows the genome location on chromosome 2 in rice. B Average nucleotide diversity ( $\pi$ ) of OsSPL4 and its flanking region ( 2 kb). C Genetic differentiation $\left(F_{S T}\right)$ across chromosome 2 between different subspecies ( Aus vs. Indica and Japonica vs. Indica). A total of 592 rice accessions were used for analysis, including 46 aus, 295 indica and 156 japonica rice from RiceVarMap database, respectively. D Haplotype network of OsSPL4 among 10 wild and 592 cultivar rice accessions. Only haplotypes found in more than 10 rice accessions were used to construct the haplotype network. Each circle represents a haplotype and circle size is proportional to the haplotype frequency. Different colors refer to different rice subpopulations. Red star corresponds to OSSPL4 ${ }^{A 92 V}$. Indl, indica I; Indll, indica II; IndInt, indica intermediate; Int, intermediate group between indica and japonica accessions; Japlnt, japonica intermediate; Nivara, O. nivara; TrJ, tropical japonica; TeJ, temperate japonica; Rufipogon, O. rufipogon. VI, intermediate group between indica and japonica/Aromatic

haplotypes based on the variation from RiceVarMap database in 529 rice accessions revealed two mainly distinct clusters: separate indica and japonica haplotype clusters (Fig. 8D). Furthermore, we also performed haplotype network analysis based on the Rice Functional Genomics and Breeding (RFGB) database, obtaining eight haplotypes for OsSPL4 (Additional file 1: Fig. S10A). One of the missense mutation SNP (C-T) at Chr02:4,074,278 (vg0204074278) in Hap 4, 5, 8 could change the Ala into Val (A92V) in the SBP domain of OsSPL4, which was identified in both RiceVarMap and RFGB database (Additional file 1: Fig. S10A and Tables S6, S7). According to the phenotypes from RFGB and RiceVarMap database, the grain length of rice varieties in Hap 5 was longer than that of Hap 1 or Hap 2, indicating that the mutation of SBP domain in the OsSPL4 could affect the grain shape (Additional file 1: Fig. S10B and Table S8).

\section{Discussion}

Although a number of grain yield-related QTLs/genes have been characterized in the past years, more yield genes are required for the high-yield rice breeding. SPL proteins are plant-specific transcription factors, which contain a SBP-box motif and participate in many crucial biological processes in plants (Guo et al. 2008). Although the biological functions and molecular mechanisms in many of the OSSPL family members have been extensively studied in rice, the underlying molecular mechanisms and regulation networks of OSSPL4 were not very clear, especially in regulating the panicle branching and grain size (Jiang et al., 2020). In this study, we generated transgenic plants by mutating of OsSPL4 using CRISPR/ Cas9 system and overexpressing of OSSPL4 to reveal its role in the regulation of panicle architecture and grain size in rice.

CRISPR/Cas9 which is a useful tool for targeted mutagenesis, can rapidly generate homozygous mutation transgenic plants in rice (Hu et al., 2020). Lately, CRISPR/ Cas-mediated base editing systems have been developed for precise base editing with reducing indels and off-target changes in plants (Molla and Yang 2019). And using CRISPR/Cas9-mediated adenine base editors (ABEs), point mutation of zebra3 and wsl 5 in rice could affect the phenotypes (Molla and Yang 2019). It has been reported that two mutation types ( $3 \mathrm{bp}$ and $39 \mathrm{bp}$ deletion) of OsIAA23 in Kasalath rice using the CRISPR/Cas9 system showed severe dwarfism, inhibited lateral root formation, reduced tiller number and exhibited lower seedsetting rate (Jiang et al. 2019). In rice, eight amino acid deletion in RGG2 (zrgg2-2) and one amino acid deletion 
in OsAOG17 (ago17-2) were also reported to regulate grain size and weight (Miao et al. 2019; Zhong et al. 2020). These results indicated that the deletion of amino acids could be resulted in alteration of agronomic traits. In the present study, two homozygous mutation (spl4- $d 3$ and spl4-d15) of OsSPL4 with depressed activity could increase grain length and width as well as grain yield, although these deletions not cause any other changes in amino acid sequences (Fig. 3, Fig. 6E, Additional file 1: Fig. S1).To further investigate the function of OsSPL4 in rice, we successfully generated transgenic plants overexpressing OSSPL4 in Nipponbare background. The OE1 and OE2 plants exhibited short panicles, decreased number of panicles and had small grains (Fig. 3; Additional file 1: Table S2). As one of the targets of OsmiR156, which targeted in the $3^{\prime}$ UTR region of OsSPL4, overexpression of osa-miR156 were found to increase the grain size in rice (Additional file 1: Figs. S6, S7). Whereas, the transgenic plants of STTM156 showed smaller grains (Additional file 1: Fig. S8).

The spl4-d3 and spl4-d15 transgenic plants, having amino acid mutations in the SBP domain of OsSPL4 protein, produced larger grains (Fig. 2). Since OsSPL4 is a plant-specific transcription factor, SBP domain is essential for binding of downstream target genes (Guo et al. 2008). Our three-dimensional structure analysis showed that the amino acids deletion of spl4- $d 3$ and spl4- $d 3$ affected the formation of zinc-binding pocket (Fig. 6A), indicating that the single valine deletion in spl4- $d 3$ might cause all effect probably by altering the binding properties of SBP domain to promoter of other downstream genes. DEP1 has been reported to be directly bound with OSSPL genes, and elevated expression level of DEP1 increased the panicle length ( $\mathrm{Lu}$ et al. 2013; Yuan et al. 2019). In our study, the expression level of DEP1 was up-regulated in the spl4-d15 transgenic lines (Additional file 1: Table S5) and the transcriptional activity of OsSPL4 were reduced in both spl4-d3 and spl4-d15 lines by the dual LUC assay (Fig. 6E).

Phylogenetic analysis reveals that OsSPL4, OsSPL11, OSSPL 3 and OsSPL12 were grouped in the same subclade, in which OsSPL11 was segmental duplication with OsSPL4 (Additional file 1: Fig. S4) (Shao et al. 2019; Xie et al. 2006). Expression patterns analysis showed that OsSPL4 was highly expressed in leaves, young panicles and developing seeds of 25DAP, indicating the roles in panicle development and grain size (Fig. 7A). Scanning electron microscopy showed that the out epidermal cells of the spikelet hulls in spl4- $d 3$ lines were much longer than that of control plants (Fig. 4A, B). And more grain number per panicle and panicle branches were observed in the spl4-d3 lines (Fig. 5A-G). The field experiments also showed that the spl4- $d 3$ lines can increase the grain yield per plant by $11.44 \%$ in Nipponbare background (Fig. 2G, Additional file 1: Table S2). RNA-seq and qRT-PCRs validated that the floral identity genes and cell-cycle genes were highly expressed in the young panicles of spl4- $d 3$ lines (Fig. 4G, Fig. 5I, Additional file 1: Table S3). Hence, the spl4- $d 3$ transgenic plants had enlarged the spikelet hulls and increased grain number, which resulted in enhancing grain size and weight as well as yield in rice (Fig. 5). Although the grain quality of OSSPL4 mutation lines have a little decrease, it could be improved by other approaches, such as crossing with high-quality sterile lines in the rice breeding ( $\mathrm{Hu}$ et al. 2015b).

We compared the expression patterns of several reported yield-related genes of rice between the control and spl4- $d 3$ transgenic plants. Co-expression network also revealed that OSSPL4 was involved in the interaction networks of different yield-related genes (Additional file 1: Fig. S3). For example, GW2 which negatively regulates grain width and weight, was down-regulated in spl4- $d 3$ lines (Additional file 1: Table S5) (Song et al. 2007). Three genes $L A X, F Z P$ and RCN1 have been shown to control the axillary meristem initiation and development, which determined the panicle branching and grain number (Huang et al. 2009). Different expression patterns of these genes were found between the spl4$d 3$ lines and control plants (Additional file 1: Table S5). Particularly, the expression level of $F Z P$ was repressed in spl4-d3 lines, which has been reported to increase the number of spikelets per panicle and grain yield (Bai et al. 2017). Several yield-related genes were significantly up-regulated in spl4- $d 3$ transgenic plants, including RFL, RGB1, GL7/GW7 and OsGIF1 (Additional file 1: Table S5). In rice, $R F L$ was reported to promote panicle branching by activating positive regulators such as $L A X$ (Rao et al. 2008). RGB1 positively regulates cellular proliferation and cell number (Utsunomiya et al. 2011). And the grain length and length of flag leaf were increased in the GL7 overexpression lines (Wang et al. 2015). As the transcription coactivator of GRF, overexpression of OsGIF1 has been reported to significantly increase grain size and weight (He et al. 2017; Li et al. 2016). Moreover, other yield-related genes such as GL3.3/TGW3, RGG2 and $O s L A C$, were significantly suppressed in the spl4$d 3$ transgenic plants (Additional file 1: Table S5). GL3.3/ TGW3 (OsSK41) and RGG2 have been demonstrated to negatively regulate grain length and weight in rice ( $\mathrm{Hu}$ et al. 2018a; Xia et al. 2018; Ying et al. 2016; Miao et al. 2019). OsLAC which is the target of OsmiR397b, is involved in the sensitivity of plants to BR. Overexpression of OsmiR397b repressed the expression of OsLAC and led to increase grain size and grain number per panicle (Zhang et al. 2013). Therefore, the mutation of OsSPL4 


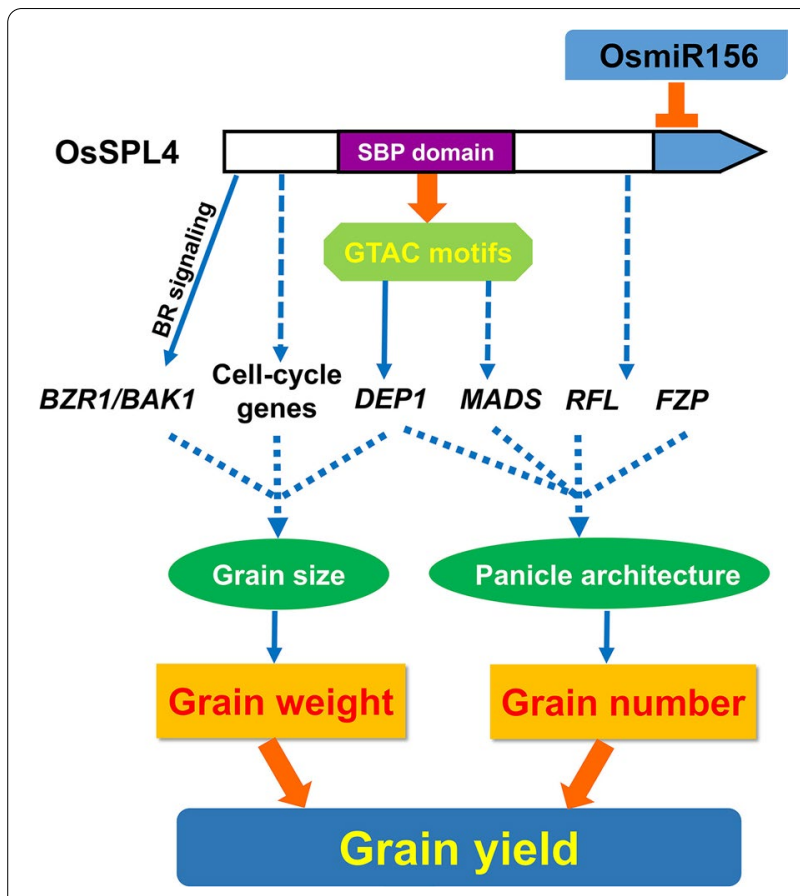

Fig. 9 Aproposed model for the regulation of grain yield by OsSPL4 in rice. Arrows indicate the positive effects and short horizontal lines represent the negative effects. The dashed lines indicate the molecular mechanisms which are unclear. BR, brassinosteroid

in this study might regulate these yield-related genes or participate in BR signaling pathway to promote panicle branching and increase grain size, resulting in high yield in rice (Fig. 9).

Compared with the homologue SPL4 genes in Oryza genus, the last exon of cultivated rice SPLs (OsSPL4 and OglaSPL4) possessed a shorter length than other wild rice SPLs (Additional file 1: Fig. S9), which is consistent with the previous report (Zhong et al. 2019). The nucleotide diversity analysis of wild and cultivated rice population suggested that OSSPL4 might have undergone artificial selection in the japonica rice (Fig. 6A). We

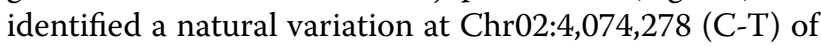
OsSPL4 among both $3 \mathrm{~K}$ and 529 rice accessions (Additional file 1: Tables S6, S7). Further editing of OsSPL4 at this site using the $\mathrm{ABE}$ system may effectively develop different grain length rice lines (Molla and Yang 2019; Molla et al., 2020). The A92V transition in the first exon, which is also located in the SBP domain, caused the change of amino acid from Ala to Val (Additional file 1: Fig. S10). This variant allele existed in the aus and indica group, which greatly contributed to grain length variance (Additional file 1: Fig. S10 and Additional file 2: Table S8). And population difference $\left(F_{\mathrm{ST}}\right)$ was also showed to be lower between aus and indica than that of indica and japonica in the OsSPL4 gene (Fig. 8C), indicating the allele in indica rice was introgressed from aus varieties under artificial selection. The results suggested that nonsense mutation occurred in the SBP domain could regulate the grain size and yield in rice.

\section{Conclusions}

We identified two elite alleles of OSSPL4, which plays an important role in the panicle and grain development of rice. Mutation of OsSPL4 in rice increased the number of grains per panicle and grain size based on the results of phenotyping, cytological observation, dual-luciferase assays, and RNA-seq analysis. And qRT-PCR also confirmed that several MADS-box and cell-cycle genes were shown to be regulated in the mutation lines. Hence, our findings not only identify OsSPL4 as a key regulator of grain size by acting on cell division control but also suggest a strategy for grain size modification in a wide range of cereal crops for yield improvement.

\section{Abbreviations}

GO: Gene ontology; QTLs: Quantitative trait loci; DBD: DNA binding domain; FDR: False discovery rate; SPL: SQUAMOSA PROMOTER BINDING PROTEIN (SBP)-like; NCBI: National Center for Biotechnology Information; CRISPR/Cas9: Clustered regularly interspaced short palindromic repeats/CRISPR-associated protein-9; STTM: Short tandem target mimic.

\section{Supplementary Information}

The online version contains supplementary material available at https://doi. org/10.1186/s12284-021-00531-7.

Additional file 1: Fig. S1. Plant phenotypes of overexpression (OE) and the mutant transgenic plants of OsSPL4. Fig. S2. Differentially expressed genes (DEGs) and GO enrichment in control and spl4-d3 plants using RNA-seq analysis. Fig. S3. Co-expression network of yield-related genes and OsSPL4 in rice. Fig. S4. Phylogenetic tree of the 19 SPL family members in rice. Fig. S5. Protein sequences and the structures in control and the mutation lines. Fig. S6. Schematic diagram of OsSPL4 as the target of OsmiR156 and the vector construction of STTM156. Fig. S7. Overexpresssion (OE) of osa-miR156 increases rice grain length and width. Fig. S8. Osa-miR156 mimicry (STTM156) transgenic rice decreases grain length and width. Fig. S9. Phylogenetic analysis and exon-intron structures of SPL4 orthologs in different Oryza genus. Fig. S10. Different haplotypes of OsSPL4. Table S1. Primers used in this study. Table S2. Grain yield and associated components in the transgenic rice in field. Table S3. Expression patterns of the floral and cell-cycle genes between control and spl4-d3 transgenic plants. Table S4. KEEG pathway of the differentially expressed genes in young panicle between spl4-d3 and control plants. Table S5. The expression patterns of rice yield-related genes between spl4-d3 and control plants. Table S6. Variations in OsSPL4 coding region identified from RFGB Database. Table S7. Variations in OsSPL4 coding region identified from RiceVarMap Database.

Additional file 2: Table S8. Phenotypes for the different haplotypes in OsSPL4 of rice.

\section{Acknowledgements}

Not applicable. 


\section{Authors' Contributions}

D.Y., W.W. and F.H. supervised the project. J.H., G.C., L.H., J.L. and Q.H. collected the samples, performed the experiments and data analysis. Y.Z., J.H., L.H., R.Z. and S.Z. constructed the vectors, performed transgenic experiments and phenotyping. H.L. carried out the haplotype analysis. J.H. G.C. and L.H. drafted the manuscript. D.Y. and W.W. revised the manuscript. All authors read and approved the final manuscript.

\section{Funding}

This work was financially supported by National Key Basic Research Program (2013CB835200), National Natural Science Foundation of China (31471464, 31901426), the Key Project of Transgenic New Varieties Culture of China (2009ZX08009-021B), Key Grants from the Yunnan Provincial Science and Technology Department (2018IC096 and 2019HC028), and Open Research Fund of State Key Laboratory of Hybrid Rice (Wuhan University) (KF201806).

\section{Availability of Data and Materials}

All the RNA-seg data were deposited in the National Center for Biotechnology Information (NCBI) under the accessions: PRJNA646823.

\section{Declarations}

Ethics Approval and Consent to Participate

Not applicable.

\section{Consent for Publication}

Not applicable.

\section{Competing interests}

The authors declare no conflicts of interest.

\section{Author details}

${ }^{1}$ State Key Laboratory of Hybrid Rice, College of Life Sciences, Wuhan University, Wuhan 430072, China. ${ }^{2}$ State Key Laboratory of Genetic Resources and Evolution, Kunming Institute of Zoology, Chinese Academy of Sciences, Kunming 650223, China. ${ }^{3}$ School of Ecology and Environment, Northwestern Polytechnical University, Xi'an 710072, China. ${ }^{4}$ State Key Laboratory for Conservation and Utilization of Bio-Resources in Yunnan, School of Agriculture, Yunnan University, Kunming 650500, China. ${ }^{5}$ Germplasm Bank of Wild Species, Kunming Institute of Botany, Chinese Academy of Sciences, Kunming 650201, China. ${ }^{6} \mathrm{BGl}-$ Baoshan, Baoshan 678004, Yunnan, China.

Received: 27 April 2021 Accepted: 16 October 2021

Published online: 02 November 2021

\section{References}

Bai XF, Wu B, Xing YZ (2012) Yield-related QTLs and their applications in rice genetic improvement. J Integr Plant Biol 5:300-311

Bai XF, Huang Y, Hu Y, Liu HY, Zhang B, Smaczniak C, Hu G, Han ZM, Xing YZ (2017) Duplication of an upstream silencer of FZP increases grain yield in rice. Nat Plants 3:885-893

Birkenbihl RP, Jach G, Saedler H, Huijser P (2005) Functional dissection of the plant-specific SBP-domain: overlap of the DNA-binding and nuclear localization domains. J Mol Biol 352:585-596

Che RH, Tong HN, Shi BH, Liu Y, Fang S, Liu D, Xiao Y, Hu B, Liu L, Wang H, Zhao M, Chu C (2015) Control of grain size and rice yield by GL2-mediated brassinosteroid responses. Nat Plants 2:15195

Dai ZY, Wang J, Yang XF, Lu H, Miao XX, Shi ZY (2018) Modulation of plant architecture by the miR156f-OsSPL7-OsGH3.8 pathway in rice. J Exp Bot 69:5117-5130

Du Z, Zhou X, Ling Y, Zhang ZH, Su Z (2010) agriGO: a GO analysis toolkit for the agricultural community. Nucleic Acids Res 38:W64-70

Gao F, Wang K, Liu Y, Chen Y, Chen P, Shi Z, Luo J, Jiang D, Fan F, Zhu Y, Li S (2015) Blocking miR396 increase rice yield by shaping inflorescence architecture. Nat Plants 2:15196
Guo AY, Zhu QH, Gu XC, Ge S, Yang J, Luo JC (2008) Genome-wide identification and evolutionary analysis of the plant specific SBP-box transcription factor family. Gene 418:1-8

He ZS, Zeng J, Ren Y, Chen D, Li W, Gao F, Cao Y, Luo T, Yuan G, Wu X, Liang Y, Deng Q, Wang S, Zheng A, Zhu J, Liu H, Wang L, Li P, Li S (2017) OsGIF1 positively regulates the sizes of stems, leaves, and grains in rice. Front Plant Sci 8:1730

Hiei Y, Ohta S, Komari T, Kumashiro T (1994) Efficient transformation of rice (Oryza sativa L.) mediated by Agrobacterium and sequence analysis of the boundaries of the T-DNA. Plant J 6:271-282

Hu B, Jin J, Guo AY, Zhang H, Luo J, Gao G (2015a) GSDS 2.0: an upgraded gene feature visualization server. Bioinformatics 31:1296-1297

Hu J, Wang YX, Fang YX, Zeng L, Xu J, Yu H, Shi Z, Pan J, Zhang D, Kang S, Zhu L, Dong G, Guo L, Zeng D, Zhang G, Xie L, Xiong G, Li J, Qian Q (2015b) A rare allele of GS2 enhances grain size and grain yield in rice. Mol Plant 8:1455-1565

Hu Z, Lu SJ, Wang MJ, He HH, Sun L, Wang H, Liu XH, Jiang L, Sun JL, Xin X, Kong W, Chu CC, Xue HW, Yang JS, Luo XJ, Liu JX (2018a) A novel QTL qTGW3 encodes the GSK3/SHAGGY-Like kinase OsGSK5/OsSK41 that interacts with OsARF4 to negatively regulate grain size and weight in rice. Mol Plant 11:736-749

Hu JH, Zeng T, Xia QM, Qian Q, Yang CD, Ding Y, Chen LN, Wang W (2018b) Unravelling miRNA regulation in yield of rice (Oryza sativa) based on differential network model. Sci Rep 8:8498

Hu JH, Zeng T, Xia QM, Huang LY, Zhang YS, Zhang CC, Zeng Y, Liu H, Zhang SL, Huang GF, Wan WT, Ding Y, Hu FY, Yang CD, Chen LN, Wang W (2020) Identification of key genes for the ultrahigh yield of rice dynamic cross-tissue network analysis. Genomics Proteomics Bioinformatics 18:256-270

Huang XZ, Qian Q, Liu ZB, Sun HY, He SY, Luo D, Xia GM, Chu CC, Li JY, Fu XD (2009) Natural variation at the DEP1 locus enhances grain yield in rice. Nat Genet 41:494-497

Huang LY, Zhang R, Huang GF, Li Y, Melaku G, Zhang S, Chen H, Zhao Y, Zhang J, Zhang Y, Hu F (2018) Developing superior alleles of yield genes in rice by artificial mutagenesis using the CRISPR/Cas9 system. Crop J 6:475-481

Ishimaru K, Hirotsu N, Madoka Y, Murakami N, Hara N, Onodera H, Kashiwagi T, Ujiie K, Shimizu B, Atsuko O, Hisashi M, Katoh E (2013) Loss of function of IAA-glucose hydrolase gene TGW6 enhances rice grain weight and increases yield. Nat Genet 45:707-711

Jiang MM, Hu HY, Kai J, Traw MB, Yang SH, Zhang XH (2019) Different knockout genotypes of OsIAA23 in rice using CRISPR/Cas9 generating different phenotypes. Plant Mol Biol 100:467-479

Jiang MM, He Y, Chen XN, Zhang XH, Guo YR, Yang SH, Huang J, Traw MB (2020) CRISPR-based assessment of genomic structure in the conserved SQUAMOSA promoter-binding-like gene clusters in rice. Plant J 104:1301-1314

Jiao YQ, Wang YH, Xue DW, Wang J, Yan M, Liu G, Dong G, Zeng D, Lu Z, Zhu X, Qian Q, Li J (2010) Regulation of OsSPL14 by OsmiR156 defines ideal plant architecture in rice. Nat Genet 42:541-544

Kumar S, Stecher G, Tamura K (2016) MEGA7: molecular evolutionary genetics analysis version 7.0 for bigger datasets. Mol Biol Evol 33:1870-1874

Lee T, Oh T, Yang S, Shin J, Hwang S, Kim C, Kim H, Shim H, Shim J, Ronald P, Lee I (2015) RiceNet v2: an improved network prioritization server for rice genes. Nucleic Acids Res 43:W122-W127

Li N, Li YH (2016) Signaling pathways of seed size control in plants. Curr Opin Plant Biol 33:23-32

Li YB, Fan CC, Xing YZ, Jiang Y, Luo L, Sun L, Shao D, Xu C, Li X, Xiao J, He Y, Zhang Q (2011) Natural variation in GS5 plays an important role in regulating grain size and yield in rice. Nat Genet 43:1266-1269

Li SC, Gao FY, Xie KL, Zeng X, Cao Y, Zeng J, He Z, Ren Y, Li W, Deng Q, Wang S, Zheng A, Zhu J, Liu H, Wang L, Li P (2016) The OsmiR396c-OsGRF4OsGIF1 regulatory module determines grain size and yield in rice. Plant Biotechnol J 14:2134-2146

Li N, Xu R, Li YH (2019) Molecular networks of seed size control in plants. Annu Rev Plant Biol 70:435-463

Liu LC, Tong HN, Xiao YH, Che RH, Xu F, Hu B, Liang CZ, Chu JF, Li JY, Chu CC (2015) Activation of Big Grain 1 significantly improves grain 
size by regulating auxin transport in rice. Proc Natl Acad Sci USA 112:11102-11107

Liu JF, Chen J, Zheng XM, Wu F, Lin Q, Heng Y, Tian P, Cheng Z, Yu X, Zhou K, Zhang X, Guo X, Wang J, Wang H, Wan J (2017) GW5 acts in the brassinosteroid signalling pathway to regulate grain width and weight in rice. Nat Plants 3:17043

Love MI, Huber W, Anders S (2014) Moderated estimation of fold change and dispersion for RNA-seq data with DESeq2. Genome Biol 15:550

Lu ZF, Yu H, Xiong GS, Wang J, Jiao YQ, Liu YH, Meng XB, Hu XM, Qian Q, Fu XD, Li JY (2013) Genome-wide binding analysis of the transcription activator IDEAL PLANT ARCHITECTURE1 reveals a complex network regulating rice plant architecture. Plant Cell 25:3743-3759

Lyu J, Huang LY, Zhang SL, He W, Zeng P, Zeng Y, Huang G, Zhang J, Ning M, Bao Y, Zhao S, Fu Q, Wade L, Chen H, Wang W, Hu F (2020) Neo-functionalization of a Teosinte branched 1 homologue mediates adaptations of upland rice. Nat Commun 11:725

Mao HL, Sun SY, Yao JL, Wang CR, Yu SB, Xu CG, Li XH, Zhang QF (2010) Linking differential domain functions of the GS3 protein to natural variation of grain size in rice. Proc Natl Acad Sci USA 107:19579-19584

Miao J, Yang ZF, Zhang DP, Wang Y, Xu M, Zhou L, Wang J, Wu S, Yao Y, Du X, Gu F, Gong Z, Gu M, Liang G, Zhou Y (2019) Mutation of RGG2, which encodes a type $B$ hetertrimeric $G$ protein $\gamma$ subunit, increases grain size and yield production in rice. Plant Biotechnol J 17:650-664

Miura K, Ikeda M, Matsubara A, Song XJ, Ito M, Asano K, Matsuoka M, Kitano H, Ashikari M (2010) OSSPL14 promotes panicle branching and higher grain productivity in rice. Nat Genet 42:545-549

Molla KA, Yang YN (2019) CRISPR/Cas-mediated base editing: technical considerations and practical applications. Trends in Biotech 37:1121-1142

Molla KA, Shih J, Yang YN (2020) Single-nucleotide editing for zebra3 and wsl5 phenotypes in rice using CRISPR/Cas9-mediated adenine base editors. aBiotech 1:106-118

Qi P, Lin YS, Song XJ, Shen JB, Huang W, Shan JX, Zhu MZ, Jiang LW, Gao JP, Lin HX (2012) The novel quantitative trait locus GL3.1 controls rice grain size and yield by regulating Cyclin-T1;3. Cell Res 22:1666-1680

Rao NN, Prasad K, Kumar PR, Vijayraghava U (2008) Distinct regulatory role for $R F L$, the rice $L F Y$ homolog, in determining flowering time and plant architecture. Proc Natl Acad Sci USA 105:3646-3651

Saeed Al, Sharov V, White J, Li J, Liang W, Bhagabati N, Braisted J, Klapa M, Currier T, Thiagarajan M, Sturn A, Snuffin M, Rezantsev A, Popov D, Ryltsov A, Kostukovich E, Borisovsky I, Liu Z, Vinsavich A, Trush V, Quackenbush J (2003) TM4: a free, open-source system for microarray data management and analysis. Biotechniques 34:374-378

Shannon P, Markiel A, Ozier O, Baliga NS, Wang JT, Ramage D, Amin N, Schwikowski B, Ideker T (2003) Cytoscape: a software environment for integrated models of biomolecular interaction networks. Genome Res 13:2498-2504

Shao YL, Zhou HZ, Wu YR, Zhang H, Lin J, Jiang X, He QJ, Zhu JS, Li Y, Yu H, Mao CZ (2019) OsSPL3, a SBP-domain protein, regulates crown root development in rice. Plant Cell 31:1257-1275

Shikata M, Koyama T, Mitsuda N, Ohme-Takagi M (2009) Arabidopsis SBP-box genes SPL10, SPL11 and SPL2 control morphological change in association with shoot maturation in the reproductive phase. Plant Cell Physiol 50:2133-2145

Si L, Chen J, Huang X, Gong H, Luo JH, Hou Q, Zhou T, Lu T, Zhu J, Shangguan Y, Chen E, Gong C, Zhao Q, Jing Y, Zhao Y, Li Y, Cui L, Fan D, Lu Y, Weng Q, Wang Y, Zhan Q, Liu K, Wei X, An K, An G, Han B (2016) OsSPL13 controls grain size in cultivated rice. Nat Genet 48:447-456

Song XJ, Huang W, Shi M, Zhu MZ, Lin HX (2007) A QTL for rice grain width and weight encodes a previously unknown RING-type E3 ubiquitin ligase. Nat Genet 39:623-630

Sun LJ, Li XJ, Fu YC, Zhu ZF, Tan LB, Liu FX, Sun XY, Sun XW, Sun CQ (2013) GS6, a member of the GRAS gene family, negatively regulates grain size in rice. J Integr Plant Biol 10:938-949

Sun PY, Zhang WH, Wang YH, He Q, Shu F, Liu H, Wang J, Wang JM, Yuan LP, Deng HF (2016) OsGRF4 controls grain shape, panicle length and seed shattering in rice. J Integr Plant Biol 10:836-847
Trapnell C, Roberts A, Goff L, Pertea G, Kim D, Kelley DR, Pimentel H, Salzberg SL, Rinn JL, Pachter L (2012) Differential gene and transcript expression analysis of RNA-seq experiments with TopHat and Cufflinks. Nat Protoc 7:562-578

Utsunomiya Y, Samejima C, Takayanagi Y, Izawa Y, Yoshida T, Sawada Y, Fujisawa $Y$, Kato H, Iwasaki $Y$ (2011) Suppression of the rice heterotrimeric $G$ protein $\beta$-subunit gene, $R G B 1$, causes dwarfism and browning of internodes and lamina joint regions. Plant J 67:907-916

Varkonyi-Gasic E, Wu R, Wood M, Walton EF, Hellens RP (2007) Protocol: a highly sensitive RT-PCR method for detection and quantification of microRNAs. Plant Methods 3:12

Wang L, Zhang QF (2017) Boosting rice yield by fine-tuning SPL gene expression. Trends Plant Sci 22:643-646

Wang SK, Wu K, Yuan QB, Liu XY, Liu ZB, Lin XY, Zeng R, Zhu H, Dong G, Qian Q, Zhang G, Fu X (2012) Control of grain size, shape and quality by OSSPL16 in rice. Nat Genet 44:950-954

Wang Y, Xiong G, Hu J, Jiang L, Yu H, Xu J, Fang Y, Zeng L, Xu E, Xu J, Ye W, Meng X, Liu R, Chen H, Jing Y, Wang Y, Zhu X, Li J, Qian Q (2015) Copy number variation at the GL7 locus contributes to grain size diversity in rice. Nat Genet 47:944-948

Wang QL, Sun AZ, Chen ST, Chen LS, Guo FQ (2018) SPL6 represses signaling outputs of ER stress in control of panicle cell death in rice. Nat Plants 4:280-288

Weng J, Gu S, Wan X, Gao H, Guo T, Su N, Lei C, Zhang X, Cheng Z, Guo X, Wang J, Jiang L, Zhai H, Wan J (2008) Isolation and initial characterization of GW5, a major QTL associated with rice grain width and weight. Cell Res 18:1199-1209

Xia D, Zhou H, Liu RJ, Dan W, Li P, Wu B, Chen J, Wang L, Gao G, Zhang Q, He Y (2018) GL3.3, a novel QTL encoding a GSK3/SHAGGY-like kinase, epistatically interacts with GS3 to produce extra-long grains in rice. Mol Plant 11:754-756

Xie KB, Wu CQ, Xiong LZ (2006) Genomic organization, differential expression, and interaction of SQUAMOSA promoter-binding-like transcription factors and microRNA156 in rice. Plant Physiol 142:280-293

Xie C, Mao XZ, Huang JJ, Ding Y, Wu JM, Dong S, Kong L, Gao G, Li CY, Wei LP (2011) KOBAS 2.0: a web server for annotation and identification of enriched pathways and diseases. Nucleic Acids Res 39:W316-W322

Yamasaki K, Kigawa T, Inoue M, Yamasaki T, Yabuki T, Aoki M, Seki E, Matsuda T, Tomo Y, Terada T, Shirouzu M, Tanaka A, Seki M, Shinozaki K, Yokoyama S (2006) An Arabidopsis SBP-domain fragment with a disrupted C-terminal zinc-binding site retains its tertiary structure. FEBS Lett 580:2109-2116

Yan J, Gu Y, Jia X, Kang W, Pan S, Tang X, Chen X, Tang G (2012) Effective small RNA destruction by the expression of a short tandem target mimic in Arabidopsis. Plant Cell 24:415-427

Yao S, Yang Z, Yang R, Huang Y, Guo G, Kong XY, Lan Y, Zhou T, Wang H, Wang WM, Cao XF, Wu J, Li Y (2019) Transcriptional regulation of miR528 by OSSPL9 orchestrates antiviral response in rice. Mol Plant 12:1114-1122

Yi R, Zhu ZX, Hu JH, Qian Q, Dai JC, Ding Y (2013) Identification and expression analysis of microRNAs at the grain filling stage in rice (Oryza sativa) via deep sequencing. PLOS ONE 8:e57863

Ying JZ, Ma M, Bai C, Huang XH, Liu LJ, Fan YY, Song XJ (2016) TGW3, a major QTL that negatively modulates grain length and weight in rice. Mol Plant 11:750-753

Yuan H, Qin P, Hu L, Zhan S, Wang S, Gao P, Li J, Jin M, Xu Z, Gao Q, Du A, Tu B, Chen W, Ma B, Wang Y, Li S (2019) OSSPL18 controls grain weight and grain number in rice. J Genet Genom 46:41-51

Zhang X, Wang J, Huang J, Lan H, Wang C, Yin C, Wu Y, Tang H, Qian Q, Li J, Zhang H (2012) Rare allele of OSPPKL1 associated with grain length causes extra-large grain and a significant yield increase in rice. Proc Natl Acad Sci USA 109:21534-21539

Zhang YC, Yu Y, Wang CY, Li Z, Liu Q, Xu J, Liao J, Wang X, Qu L, Chen F, Xin P, Yan C, Chu J, Li H, Chen Y (2013) Overexpression of microRNA OsmiR397 improves rice yield by increasing grain size and promoting panicle branching. Nat Biotechnol 31:848-852

Zhao H, Yao W, Ouyang YD, Yang WN, Wang GW, Lian XM, Xing YZ, Chen LL, Xie WB (2015) RiceVarMap: a comprehensive database of rice genomic variations. Nucleic Acids Res 43:D1018-D1022 
Zheng TQ, Yu H, Zhang HL, Wu Z, Wang W, Tai S, Lu C, Ran J, Wei C, Shi J, Gao Y, Fu B, Zhou Y, Zhao X, Zhang F, MCNALLY K, Li Z, Zhang G, Li J, Zhang D, Xu J, Li ZK (2015) Rice functional genomics and breeding database (RFGB)3K-rice SNP and InDel sub-database. Sci Bull 60:367-371

Zhong H, Kong WL, Gong ZY, Fang XY, Deng XX, Liu C, Li YS (2019) Evolutionary analyses reveal diverged patterns of SQUAMOSA promoter binding protein-like (SPL) gene family in Oryza genus. Front in Plant Sci 10:565

Zhong J, He W, Peng Z, Zhang H, Li F, Yao J (2020) A putative AGO protein, OsAGO17, positively regulates grain size and grain weight through OsmiR397b in rice. Plant Biotechnol J 18:916-928

Zhou Y, Zhu J, Li Z, Yi C, Liu J, Zhang H, Tang S, Gu M, Liang G (2009) Deletion in a quantitative trait gene QPE9-1 associated with panicle erectness improves plant architecture during rice domestication. Genetics 183:315-324

Zhu ZF, Tan LB, Fu YC, Liu FX, Cai HW, Xie DX, Wu F, Wu JZ, Matsumoto T, Sun CQ (2013) Genetic control of inflorescence architecture during rice domestication. Nat Commun 4:2200

Zuo J, Li J (2014) Molecular genetic dissection of quantitative trait loci regulating rice grain size. Annu Rev Genet 48:99-118

\section{Publisher's Note}

Springer Nature remains neutral with regard to jurisdictional claims in published maps and institutional affiliations.

\section{Submit your manuscript to a SpringerOpen ${ }^{\circ}$ journal and benefit from:}

- Convenient online submission

- Rigorous peer review

- Open access: articles freely available online

- High visibility within the field

- Retaining the copyright to your article

Submit your next manuscript at springeropen.com 